\title{
Learn to Live Longer: Counterfactual Inference using Balanced Representations for Parametric Deep Survival Analysis
}

This paper was downloaded from TechRxiv (https://www.techrxiv.org).

LICENSE

CC BY 4.0

SUBMISSION DATE / POSTED DATE

02-02-2022 / 10-02-2022

\section{CITATION}

Prasad, Ranjitha (2022): Learn to Live Longer: Counterfactual Inference using Balanced Representations for Parametric Deep Survival Analysis. TechRxiv. Preprint. https://doi.org/10.36227/techrxiv.19105451.v1

DOI

10.36227/techrxiv.19105451.v1 


\title{
Learn to Live Longer: Counterfactual Inference using Balanced Representations for Parametric Deep Survival Analysis
}

\author{
Muskan Gupta, Ranjitha Prasad Member, IEEE, and Garima Gupta
}

\begin{abstract}
Learning causal relationships among entities is vital in healthcare, especially for clinical decision making and diagnosis. In this work, we design novel frameworks for counterfactual inference $(\mathrm{Cl})$ for time-to-event data. Counterfactual inference involves obtaining the average treatment effects on factual outcomes relative to the counterfactual outcomes. In the context of time-to-event data, our goal is to reliably estimate the average treatment effect in terms of time-to-event, on the observed (factual) outcomes as compared to the unobserved counterfactual population. In order to analyse time-to-event, we model the survival or hazard function of time-to-event, in the presence of censoring. Due to the nature of observational data, this framework is prone to treatment selection bias and censoring bias (random or instance-dependent). Using deep neural networks, we propose SurvCI and SurvCI-Info frameworks which incorporate balanced representations and treatment-specific log-time regression model for time-toevent prediction. In particular, we derive the evidence lower bound (ELBO) frameworks for learning a log-time mixture model while incorporating binary treatments and both, noninformative and informative censoring. We demonstrate the performance of proposed models using survival and causal metrics, on a synthetic, ACTG semi-synthetic and the realworld Metabric datasets, and compare it with the state-ofthe-art benchmarks.
\end{abstract}

\section{INTRODUCTION}

Over the past few years, the primary interest in significant number of machine learning (ML) applications involve evaluating the cause-effect relationship between interventions (or treatments) and outcomes, especially in economics, retail, healthcare and several other safety-critical domains [1]-[4]. This has lead to the tremendous growth of the domain of causal inference which encompasses a set of mathematical tools for estimating the effects of treatments on continuous (or binary) end-points. We consider the popular potential outcomes framework [5] where the goal is to obtain relative impacts of treatments while employing the counterfactual inference (CI) framework in order to address the absence of all possible potential outcomes for a given treatment [6].

Traditionally, randomized controlled trials (RCT) are considered as the gold standard in generating evidence about

This manuscript was first submitted on Feb. 1, 2022

Muskan Gupta is with TCS Innovation Labs, Delhi (e-mail: muskan.gupta4@tcs.com) and this work was done as a student at IIID.

Ranjitha Prasad is with Indraprastha Institute of Information Technology, Delhi (IIITD) (e-mail: ranjitha@iiitd.ac.in).

Garima Gupta is with TCS Innovation Labs, Delhi (e-mail: garima.gupta1@tcs.com) the efficacy of a treatment. However, in practice, RCTs are expensive, resulting datasets are small, and they entail ethical and legal constraints. We focus on observational data, where data is abundant, and hence, it allows for the estimation of heterogeneous causal effects [7]. However, unlike data in RCT settings, observational data is characterized by confounding, where covariates affect both, the treatments and the outcome, resulting in selection bias, as depicted in Fig. 1. Mitigating confounding effect is crucial for evaluating unbiased treatment effects [8].

Often, certain datasets constitutes treatments that have an effect on the time-to a pre-defined event. For instance, in the advertising framework a relevant study asks the question, Will an advertisement have an effect on the individuals' time-tonext-purchase? [9]. Here, the pre-defined event is purchase, and the treatment is posting the advertisement. For timeto-event modeling, we employ survival analysis (SA) which comprises of a set of statistical methods for analyzing the time-to-event (or survival time) [10]. A notable drawback of SA datasets is the issue of right-censoring, where time-toevent for individuals may not be available as they drop out of the study prior to the occurrence of the event. In this work, we are interested in evaluating the causal effect of an intervention or treatment on survival time in the presence of censoring, as depicted in Fig. 1. Since the outcome is timeto-event (non-negative), statistical assumptions employed in CI literature with real [6] or binary outcomes are not readily applicable. Further, existing CI models do not handle the issue of censoring. On the other hand, existing SA models handle random (non-informative) covariate-independent censoring effectively [11], but do not handle covariate-dependent informative censoring.

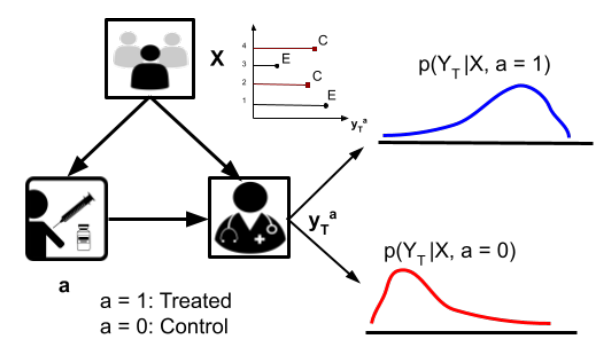

Fig. 1. Causal survival framework in observational data: The covariates $\mathbf{X}$ are confounding as they affect both, treatment $\boldsymbol{a}$ and survival time $\boldsymbol{y}_{T}^{a}$. Typical SA data consists of events (E) and censored (C) observations. Each treatment leads to a different survival distribution per-instance. 
Contributions: We propose a framework for balanced counterfactual inference from observational data, when the outcome is time-to-event. In the process, we compensate for the confounding bias and informative or non-informative censoring. Our contributions are as follows:

- We propose the SurvCI model for CI in survival datasets in the presence of non-informative (or random) censoring. We bridge the link between SA and CI by employing restricted mean survival time (RMST) in the loss function, allowing us to learn from both, event and censored data towards CI. We rigorously derive a novel, binary treatment based evidence lower bound (ELBO) for parametric SA and design a deep neural network (DNN) for learning the per-individual parameters.

- We propose the SurvCI-Info model for CI in survival datasets in the presence of informative censoring. Towards this model, our contributions are as follows:

- We extend the DSM framework [12] to handle informative censoring, where, we model time-tocensoring and rigorously derive an ELBO-based loss for SA in the presence of covariate dependent (informative) censoring.

- We extend the SurvCI model to consider covariate dependent time-to-censoring alongside time-toevent modeling. We we propose the SurvCI-Info model which incorporates a novel time-to-censoring based ELBO framework. We design a DNN for learning the per-instance parameters for predicting both, time-to-censoring and time-to-event.

We use survival metrics such as concordance index (C-Index) and hazard rate, and causal metrics such as ATE and precision in estimation of heterogeneous effect (PEHE) to evaluate the performance of the proposed methods. We demonstrate the performance of the proposed techniques on a novel synthetic and ACTG semi-synthetic datasets with random and informative censoring, and the real-world Metabric dataset. We show that the proposed methods outperform survival baselines such as DSM [12], causal baselines such as CFRNet [6] and stateof-the-art causal survival baselines based on continuous time survival prediction such as CSA.

\section{Related Works and Novelty}

Statistical tools for CI and SA have been extensively developed over the last few decades. Owing to the vast amounts of data available, DNN based advanced ML models have been developed recently. Despite these advancements, very few approaches have been proposed for CI in the context of timeto-event modeling, albeit being a very crucial aspect in several practical applications.

SA based techniques can be classified as discrete-time (DT-SA) and continuous-time (CT-SA) models. In discretetime models time-to-event is discretized, and hence, its prediction is effectively a multi-class classification problem. In continuous-time models, estimation of time-to-event or survival/hazard density is a problem of regression. Continuoustime models include Cox proportional hazard ( $\mathrm{PH})$ [13] and Accelerated failure time (AFT) models [14]. Modern CT-SA method namely DeepSurv is based on the Cox PH model and incorporates a multi-layer perceptron for learning survival time distribution [15], [16]. Both Cox model and Deepsurv incorporate the $\mathrm{PH}$ assumption which is not satisfied by most datasets. Techniques that do not use the PH assumption include parametric deep learning techniques [17], tree based methods [18], and a popular discrete-time method known as DeepHit [19]. Our work is closely related to deep survival machines (DSM), which is a continuous-time parametric mixture-model approach for time-to-event prediction [12]. DSM is the stateof-the-art method for SA, and it outperforms benchmarks such as DeepSurv and DeepHit. Note that all of the above SA models address the issue of random censoring, but not informative or instance-dependent censoring [20], [21].

Another important facet of our work is counterfactual inference, where the primary goal is to uncover the cause and effect relationship between treatments and outcomes. Confounding features that affect both treatments and outcomes are difficult to handle since they do not allow unbiased estimation of treatment effects via ATE. Recent DNN-based approaches for CI include a latent variable counterfactual distribution modeling [22], generative adversarial networks (GANs) [23] and variational autoencoders [24], [25], etc. Our work is closely related to CFRNet, which learns the balanced representation to minimize selection bias [6], [26]-[28]. Although SA and CI have been handled individually, very few papers propose methods that handle treatment estimation in SA based observational datasets. In the context of RCT, some works investigate the impact of censoring in causal SA [29], cater to selection bias in SA using probability weighting and forest based methods [30]-[32]. Such models are incapable of handling observational high-dimensional data and complex interactions among covariates.

The recently proposed continuous-time SA based counterfactual survival analysis (CSA) [33] and discrete-time SA based SurvITE [34] are the only techniques for heterogeneous and personalized CI in SA datasets. Both these models optimize the average (heterogeneous) treatment effect via counterfactual prediction of survival outcomes in observational studies, while leveraging on the balanced representation learning [6]. While CSA uses a non-parametric adversarial model [35] for SA, SurvITE uses a hazards prediction model.

We present a table that quickly compares the capabilities of the prominent DNN-based SA and CI algorithms available in the literature. Primarily, DeepHit, DeepSurv and DSM are algorithms for SA, with varying set of assumptions. In particular, we point out the methods that use the $\mathrm{PH}$ assumption as SA-PH. CFRNet and TARNet are primarily methods for CI. Further, SA-CI refers to algorithms capable of CI in SA datasets. Existing methods include CSA, CSA-info and SurvITE which can handle datasets with both, random and informative censoring (I-SA). CSA is continuous-time based, while SurvITE is discrete-time SA (DT-SA) based method. In comparison, the proposed methods namely SurvCI and SurvCI-Info is a parametric generative modeling approach for CI and CT-SA in the presence of random and informative censoring.

Novelty and Significance: As alluded to earlier, the chal- 


\begin{tabular}{|c||c|c|c|c|c|c|}
\hline Algorithm & SA & SA-PH & CI & SA-CI & I-SA & DT-SA \\
\hline \hline DSM & $\checkmark$ & $\times$ & $\times$ & $\times$ & $\times$ & $\times$ \\
\hline CRFNet & $\times$ & $\times$ & $\checkmark$ & $\times$ & $\times$ & - \\
\hline TARNet & $\times$ & $\times$ & $\checkmark$ & $\times$ & $\times$ & - \\
\hline DeepHit & $\checkmark$ & $\times$ & $\times$ & $\times$ & $\times$ & $\checkmark$ \\
\hline DeepSurv & $\checkmark$ & $\checkmark$ & $\times$ & $\times$ & $\times$ & $\times$ \\
\hline CSA & $\checkmark$ & $\times$ & $\checkmark$ & $\checkmark$ & $\checkmark$ & $\checkmark$ \\
\hline SurvITE & $\checkmark$ & $\times$ & $\checkmark$ & $\checkmark$ & $\checkmark$ & $\checkmark$ \\
\hline SurvCI & $\checkmark$ & $\times$ & $\checkmark$ & $\checkmark$ & $\checkmark$ & $\times$ \\
\hline
\end{tabular}

TABLE I

COMPARISON OF CAPABILITIES EXISTING MODELS FOR SURVIVAL AND COUNTERFACTUAL ANALYSIS

lenges in CI for survival data includes censoring bias, which may be informative (covariate-dependent) or non-informative (random). Intuitively, estimating treatment effects from timeto-event data where outcome lies in a time-interval is more involved as compared to estimating treatment effects in the standard single time-step-type setup. That is, unlike in the conventional CI scenario [6] where estimation of treatment effects is performed for a single time-step, one needs to consider the treatment response of the population over the entire time-to-event interval $\left(\left[0, T^{*}\right]\right)$. However, the framework of SA helps us to tie the estimation problem over the entire timeinterval. We show that the average treatment is interpreted as the difference in the area under the survival curves, also known as the difference in RMST for survival data. This ensures that target populations at different time-to-event epochs are associated to the same overall survival function. This approach is favored as against estimation of different survival functions at each time-step, since merging different survival functions to provide a single predicted survival (or hazard) function becomes a daunting step.

In this work, we propose SurvCI and SurvCI-Info models where the survival function is a parametric mixture model of primitive distributions such as log-normal or Weibull. Mixture models are powerful modeling techniques with vast expressibility, while parametric models are simpler to interpret [11], [12]. In the non-informative censoring framework both event and censored observations are assumed to be sampled from a single survival density, and the estimated survival density is employed in ATE estimation via RMST. On the other hand, in the informative censoring framework, we learn two different survival densities, for time-to-event and timeto-censoring. Both these densities are employed in ATE estimation via RMST. In contrast, CSA [36] and SurvCI only incorporate event data in the ATE estimation. Finally, as noted by [34], DT-SA models such as SurvITE are prone to covariate shifts due to representation of data in long format. As mentioned in [34], log-time AFT based survival models do not suffer from the issue of covariate shifts.

\section{Preliminaries and Problem Formulation}

In this section, we describe the dataset and the notations used in this work. Subsequently, we define the ELBO framework for SA [12] and the balanced representation framework for CI [6] along with the assumptions in each framework.

Let $\mathcal{D}$ be the observational dataset with $N$ samples. The $i$ th sample is represented as $\left\{\mathbf{x}_{i}, a_{i}, \delta_{i}, y_{i}\right\}$. Here, $\mathbf{x}_{i} \in \mathbb{R}^{D}$ denotes the covariates of the $i$-th instance and $\mathbf{x}_{i} \in \mathcal{X}$. We assume that $\mathbf{x}_{i} \sim \mathbf{X}_{i}$ is the set of covariates for the $i$-th individual, where $\mathbf{X}_{i}$ represents a multi-variate random variable. We assume that individual receives binary treatment $a_{i} \in\{0,1\}$, and the time-to-event outcome $y_{i}^{a}$ is observed where $y_{i}^{a} \sim Y_{T}$. Here, $Y_{T}$ is a random variable with support on the interval $\left[0, T^{*}\right]$, where $T^{*}$ is the maximum time-epoch in $\mathcal{D}$. We assume that only one treatment is provided to an individual $i$ at any given point in time, and hence, both factual and counterfactual outcomes are not available for any individual, i.e., we observe either $y_{i}^{1}$ or $y_{i}^{0}$. If $\delta_{i}=1$, it indicates that the event of interest occurs (uncensored) and $y_{i}^{a}=y_{T, i}^{a}$ is the outcome for the treatment $a_{i}$, i.e., $y_{T, i}^{0}$ is the observed time-to-event if $a_{i}=0$, and $y_{T, i}^{1}$ is the observed time-to-event if $a_{i}=1$. If $\delta_{i}=0$, the sample is rightcensored, and time-to-event is not observed. We assume that in the presence of censoring, the time for which a participant survived in the study is time-to-censoring, i.e., $y_{i}^{a} \sim Y_{C}$ and $y_{i}^{a}=y_{C, i}^{a}$. Evidently, for any given individual $i$, we observe either time-to-event $y_{T, i}^{a}$ or time-to-censoring $y_{C, i}^{a}$ irrespective of the treatment. We model two censoring scenarios in our work, (a) conditionally independent or informative censoring: $Y_{T} \Perp Y_{C} \mid \mathbf{X}$ and (b) random or non-informative censoring: $Y_{T} \Perp Y_{C}[36] .^{1}$

\section{A. Survival analysis and Deep Survival Machine}

For an observational dataset, survival function and hazard function are the most important statistics in the context of time-to-event modeling [10]. The survival function $S(\cdot)$ is the probability that an individual survives beyond a particular duration of time, and it is a monotonically decreasing function - We consider binary treatments for any individual $i$, i.e., survival function for $a=1$ given by $S^{1}\left(y_{i}^{a} \mid \mathbf{x}_{i}\right)$ is distinct as compared to the survival function for $a=0$, given by $S^{0}\left(y_{i}^{a} \mid \mathbf{x}_{i}\right)$ for all $i$. Hazard function is defined as the instantaneous probability of the event (of interest) occurring between time $\left[y_{i}^{a}, y_{i}^{a}+\Delta\right]$, given that the event has not been observed yet. We use the notion of $i$-th user specific hazard function per treatment, represented as $h\left(y_{i}^{a} \mid \mathbf{x}_{i}\right)$, given as

$$
h\left(y_{i}^{a} \mid \mathbf{x}_{i}\right)=\lim _{\Delta \rightarrow 0} \frac{P\left(y_{i}^{a} \leq Y_{T} \leq y_{i}^{a}+\Delta \mid Y_{T}>y_{i}^{a}, \mathbf{x}_{i}\right)}{\Delta}=\frac{f\left(y_{i}^{a} \mid \mathbf{x}_{i}\right)}{S^{a}\left(y_{i}^{a} \mid \mathbf{x}_{i}\right)},
$$

where $f\left(y_{i}^{a} \mid \mathbf{x}_{i}\right)$ denotes the probability density function (pdf) or failure density of time-to-event. Albeit the difference in semantic interpretation, definitions of survival time and hazard time are applicable to time-to-censoring as well. In the sequel, we describe the mixture-model based SA and balanced CI methods employed in this work.

\section{B. Deep Survival Machine (DSM)}

In this section we describe DSM which is a parametric SA technique [12] based on generative modeling, where the survival function is a weighted mixture of $K$ well-defined

\footnotetext{
${ }^{1}$ There are other definitions of informative censoring in the statistics literature. However, for the problem to be identifiable, we use the assumption [20] that given user covariates, survival time and censoring time are independent, i.e., $Y_{T} \Perp Y_{C} \mid \mathbf{X}$
} 
parametric survival distributions. Unlike Cox regression and DeepSurv [15], DSM does not employ the PH assumption. DSM is a CT-SA technique that outperforms both, DeepSurv and DeepHit [19], demonstrating robust performance of the model at different quantiles of event times in the presence of varying extents of censoring [12].

The generative modeling strategy in DSM is as follows:

- The covariates of an individual $i$ are sampled from the dataset, i.e., $\mathbf{x}_{i} \sim \mathcal{D}$.

- The parameters of the model are drawn from a zero mean Gaussian distribution, i.e., $\mathbf{w}, \zeta, \xi \sim \mathcal{N}(0,1 / \lambda)$.

- The latent variable $z_{i}$ is drawn conditioned on $\mathbf{x}_{i}$ and $\mathbf{w}_{i}$ such that $z_{i} \sim \operatorname{Discrete}\left(\sigma_{k}\left(\psi_{\Theta}\left(\mathbf{x}_{i}\right)^{T} \mathbf{w}_{i}\right)\right)$, where $\psi(\cdot)$ is an MLP with learnable parameters $\Theta$.

- The parameters $\tilde{\beta}_{k} \sim \mathcal{N}\left(\beta_{0}, 1 / \lambda\right)$ and $\tilde{\eta}_{k} \sim \mathcal{N}\left(\eta_{0}, 1 / \lambda\right)$ for all $k$, where $\beta_{0}, \eta_{0}, \lambda$ are prior parameters.

- The time to event $y_{T, i} \sim \operatorname{PRIMITIVE}\left(\beta_{k}, \eta_{k}\right)$ where,

$$
\begin{aligned}
\beta_{k} & =\tilde{\beta}_{k}+\operatorname{act}\left(\psi_{\Theta}\left(\mathbf{x}_{i}\right)^{T} \zeta\right) \\
\eta_{k} & =\tilde{\eta}_{k}+\operatorname{act}\left(\psi_{\Theta}\left(\mathbf{x}_{i}\right)^{T} \xi\right),
\end{aligned}
$$

In the above, act $(\cdot)$ is the SELU or Tanh activation functions for the Weibull or Log-Normal distribution, respectively. The above parameterization results in $\{\Theta, \xi, \zeta, \beta, \eta, \mathbf{w}\}$ being the parameters to be learnt during training, where $\mathrm{w}$ correspond to the mixture weights. In the above, PRIMITIVE $(\cdot)$ represents Weibull or Log-normal distribution, which are common in log-time based regression models since the outcome is timeto-event. Hence, the final personalised survival distribution for the event time $y_{T, i}$ is a weighted average of the failure (or survival) distributions over the $K$ mixture distributions. The component distributions are Weibull or Log-Normal, with shape and scale parameters given by $\beta_{k}$ and $\eta_{k}$ for $k=$ $1, \ldots, K$. A key aspect of the formulation is the modeling of the latent variable $Z_{i}$ which characterizes the mixture density. It is assumed that the probability of the sample $\mathbf{x}_{i}$ being sampled from the mixing component $Z_{i}=k_{i}$ is given by $P\left(Z_{i}=k_{i} \mid \mathbf{X}_{i}=\mathbf{x}_{i}\right)=\sigma_{k}\left(\psi_{\Theta}\left(\mathbf{x}_{i}\right)\right)$, where $\sigma_{k}\left(\psi_{\Theta}\left(\mathbf{x}_{i}\right)\right)$ is the softmax function considered over the image of $\mathbf{x}$ under the representation $\psi_{\Theta}(\cdot)$. The loss function to be optimized in DSM is written as

$$
\mathcal{L}_{\text {surv }}=\mathcal{L}_{U}+\alpha \mathcal{L}_{C}+\mathcal{L}_{\text {prior }},
$$

where

$$
\begin{aligned}
& \mathcal{L}_{U}=\sum_{i=1}^{|\mathcal{D}|} \mathbb{E}_{Z_{i} \sim\left(\cdot \mid \mathbf{x}_{i}, \mathbf{w}_{i}\right)}\left[\log P\left(Y_{T}=y_{T, i} \mid Z_{i}, \beta_{k}, \eta_{k}\right)\right], \\
& \mathcal{L}_{C}=\sum_{i=1}^{|\mathcal{D}|} \mathbb{E}_{Z_{i} \sim\left(\cdot \mid \mathbf{x}_{i}, \mathbf{w}_{i}\right)}\left[\log P\left(Y_{T}>y_{T, i} \mid Z_{i}, \beta_{k}, \eta_{k}\right)\right] .
\end{aligned}
$$

In the above, the $\mathbb{E}_{Z_{i} \sim\left(\cdot \mid \mathbf{x}_{i}, \mathbf{w}_{i}\right)}[\cdot]=\sigma_{K}(\psi(\cdot))$, and hence, $\mathcal{L}_{U}=\sum_{i=1}^{|\mathcal{D}|} \sigma_{K}\left(f\left(y_{T, i} \mid \beta_{k_{i}}, \eta_{k_{i}}\right)\right)$, where $f(\cdot)$ denotes the failure density. Further we write $\mathcal{L}_{C}=\sum_{i=1}^{|\mathcal{D}|} \sigma_{K}\left(S\left(y_{T, i} \mid \beta_{k_{i}}, \eta_{k_{i}}\right)\right)$, where $S(\cdot)$ denotes the survival density of $y_{T, i}$. For the purposes of brevity, in the final expressions for $\mathcal{L}_{U}$ and $\mathcal{L}_{C}$, we omit $Z_{i}$ and instead use $\beta_{k_{i}}$ and $\eta_{k_{i}}$ as the final personalized mixture weights per individual. In (3), $\alpha$ is a scalar hyperparameter that trades off the loss from uncensored and randomly censored observations, and intuitively, it provides the effect of diminishing bias arising from the long tails of survival distribution. Note that $\mathcal{L}_{\text {prior }}$ is given by

$$
\begin{aligned}
\mathcal{L}_{\text {prior }} & =\log \left(\prod_{k=1}^{K} P\left(\beta_{k}, \eta_{k} \mid \beta_{0}, \eta_{0}\right)\right) \\
& =\lambda \sum_{k=1}^{K}\left\|\beta_{k}-\beta_{0}\right\|_{2}^{2}+\left\|\eta_{k}-\eta_{0}\right\|_{2}^{2},
\end{aligned}
$$

where $\beta_{0}$ and $\eta_{0}$ are prior parameters.

In the next section, we introduce CFRNet, a DNN-based balanced representation method for CI.

\section{Balanced Counterfactual Analysis}

In this section, we discuss the CFRNet framework for predicting ATE from observational data. This framework learns a balanced representation which renders treated and control distributions similar to those as in the RCT setting. In particular, it is theoretically shown that the imbalance in control and treated distribution is upper-bounded using an Integral Probability Metric (IPM) [6]. The assumptions used in this work (and in ours) are as follows:

Assumption 1 (Conditional ignorability and positivity): These assumptions state that the potential outcomes are independent of the treatment assignment conditional on the covariates (unconfoundedness), i.e., $y_{i}^{1}, y_{i}^{0} \Perp a \mid \mathbf{x}_{i}$, and that every individual has some positive probability to be assigned to each treatment, i.e., $0<p\left(a \mid \mathbf{x}_{i}\right)<1, \forall a$ [37].

Assumption 2 (SUTVA): Under this assumption, we say that there are no hidden treatments, and the treatment of $i$-th individual does not affect the potential outcomes of the $j$-th individual, where $i \neq j$. [38].

Estimands of Interest: The average treatment effect (ATE) is an estimand of interest, and it is defined as

$$
\tau(\mathbf{x}) \triangleq \mathbb{E}_{\mathbf{X}}\left[y_{i}^{1}-y_{i}^{0} \mid \mathbf{x}_{i}\right] .
$$

CFRNet uses a loss function that minimizes the expected squared error (SE) between the true $(\tau(\mathbf{x}))$ and predicted ATE $(\hat{\tau}(\mathbf{x}))$. This metric is also called as Precision in Estimation of Heterogeneous Effect (PEHE) and is given by:

$$
\epsilon_{P E H E}=\int_{\mathcal{X}}(\hat{\tau}(\mathbf{x})-\tau(\mathbf{x}))^{2} p(\mathbf{x}) d \mathbf{x} .
$$

Loss Function: The authors in [6] theoretically show that in order to optimize PEHE error and learn the parameters in the representation and the hypothesis layers, the following loss function is optimized:

$$
\begin{aligned}
\mathcal{L}_{C I}=\frac{\beta^{\prime}}{N} \sum_{i=1}^{N} v_{i} L\left(h\left(\Phi\left(\mathbf{x}_{i}, a_{i}\right)\right), y_{i}^{a}\right)+\lambda^{\prime} R(h) & \\
& +\kappa \operatorname{IPM}\left(\mu\left(\Phi\left(\mathbf{x}_{i}, a=1\right)\right), \mu\left(\Phi\left(\mathbf{x}_{i}, a=0\right)\right)\right),
\end{aligned}
$$

where $\left\{\beta^{\prime}, \lambda^{\prime}, \kappa\right\}$ are the hyperprameters, $v_{i}=\frac{a}{2 s}+\frac{(1-a)}{2(1-s)}$, $a_{i}$ represents the factual treatment, $R(\cdot)$ is a model complexity term, $s=\frac{1}{N} \sum_{i=1}^{N} t_{i}$ and $L(\cdot, \cdot)$ is the SE loss,i.e., $\left.L\left(h\left(\Phi\left(\mathbf{x}_{i}, a\right)\right), y_{i}^{a}\right)=\| h\left(\Phi\left(\mathbf{x}_{i}, a\right)\right)-y_{i}^{a}\right) \|_{2}^{2}$. Further, we use the Maximum Mean Discrepancy (MMD) in IPM [39] and accordingly, $\mu\left(\Phi\left(\mathbf{x}_{i}, a\right)\right)$ is obtained as given in [6]. The hypotheses layer $h\left(\Phi\left(\mathbf{x}_{i}, a\right)\right)$ is designed as two separate branches, one for $a=1$ and the other for $a=0$. During 
training, each sample is used to update only the branch corresponding to the observed treatment. Note that $\kappa$ is a hyperparameter that signifies the importance of the IPM term in (8). In the RCT setting the IPM term is 0. Accordingly, TARNet is a well-known variant of CFRNet when $\kappa=0$ [6]. In the context of survival analysis, $\mathcal{L}_{C I}$ (and in particular $\left.L\left(h\left(\Phi\left(\mathbf{x}_{i}, a\right)\right), y_{i}^{a}\right)\right)$ is computed over the $N$ observations which consists of both, event and censored observations. However, since time-to-event is not available for censored observations, existing approaches append another function to handle censored observations [36]. In contrast, our approach is to interpret $L(\cdot, \cdot)$ in such a way that it utilizes both, timeto-event outcomes and censored observations, allowing us to continue with the basic framework of CFRNet, and hence inculcating the theoretical guarantees in CFRNet.

\section{Survei for Non-Informative Censored DatA}

In this section, we propose the SurvCI (Survival analysis based Counterfactual Inference) framework in the presence of non-informative (random) censoring. We derive the parametric ELBO for the mixture model based SA in the presence of binary treatments followed by a novel loss function for CI which incorporates both, time-to-event and censored observations. In particular, we utilize the relationship between ATE and RMST as given in Sec. IV-A and propose to modify $L(\cdot, \cdot)$ (which is the SE term in (8)) in such a way that it utilizes both, timeto-event outcomes and censored observations.

\section{A. Restricted Mean Survival-Time}

We utilize the well-known result that relates ATE to the area under the survival distribution for a given treatment, also known as restricted mean survival time (RMST) [40]. The following proposition states this relationship by utilizing the results on the expectation of a random variable with nonnegative support.

Proposition 1: For the survival data given in Sec. III, ATE in (6) is given by

$$
\tau(\mathbf{x})=\int_{0}^{\infty} S^{1}\left(y_{i}^{a} \mid \mathbf{x}\right) d y-\int_{0}^{\infty} S^{0}\left(y_{i}^{a} \mid \mathbf{x}\right) d y .
$$

Proof: For a random variable $W$ with non-negative support, the following property holds:

$$
\mathbb{E}_{W}[w]=\int_{0}^{\infty}(1-F(w)) d w=\int_{0}^{\infty} S(w) d w,
$$

where $F(w)$ represents the cdf of $W$, and $S(w)=(1-F(w))$ is the survival distribution, as defined in the previous section. Since time-to-event $Y$ is a non-negative random variable, we apply the above result for a given instance $\mathbf{x}_{i}$ subject to treatment $a$ to obtain

$$
\mathbb{E}\left[y_{i}^{a} \mid \mathbf{x}_{i}, a\right]=\int_{0}^{\infty} S^{a}\left(y_{i}^{a} \mid \mathbf{x}_{i}\right) d y,
$$

where $S^{a}\left(\cdot \mid \mathbf{x}_{i}\right)$ represents the $i$-th instance specific survival function. Using (11), we can re-write (6) as given in (9).

The form of ATE given in (9) suggests that area under the survival curve is a good estimate of survival time, under a given treatment. This is not surprising as in statistical theory of survival analysis, the area under the survival curve is known as Restricted Mean Survival Time and has been used to estimate survival time [40], [41]. In particular, when RMST per treatment is used in $\epsilon_{P E H E}$, then the PEHE metric measures the variance in survival time.

It is impractical to compute (9) for an infinite duration, and hence, the upper limit of the integral above is restricted to maximum time-horizon, $T^{*}$. The estimated treatment effect estimate for unit $\mathbf{x}$ is given by:

$$
\hat{\tau}(\mathbf{x})=\hat{y}_{i}^{1}-\hat{y}_{i}^{0}=\int_{0}^{T^{*}} \hat{S}^{1}\left(y_{i}^{1} \mid \mathbf{x}\right) d y_{i}-\int_{0}^{T^{*}} \hat{S}^{0}\left(y_{i}^{0} \mid \mathbf{x}\right) d y_{i},
$$

where, $\hat{S}^{1}(\cdot)$ and $\hat{S}^{0}(\cdot)$ is learnt from data. When $y_{i}^{a}=y_{T, i}^{a}$, it represents time-to-event which is the time at which the event occurred. On the other hand, when $y_{i}^{a}=y_{C, i}^{a}$, it represents time-to-censoring, which is the time for which the individual survived in the study before being censored. Since survival function is a statistic that incorporates both these aspects, it is imperative that $L(\cdot, \cdot)$ is based on the survival function.

\section{B. Proposed SurvCI Framework}

In this section, we derive the ELBO for SA with binary treatments. The framework described in Sec. III-A utilizes the parametric mixture density formulation for predicting the time-to-event. Using the following theorem, we enable this parametric framework to handle the binary treatment scenario estimating distinct survival functions for treated and control, where each survival function is a weighted mixture of $K$ well defined parametric survival distributions.

Theorem 1: The overall ELBO-based loss function for the survival dataset $\mathcal{D}$ defined in Sec. III in the presence of binary treatments is given by

$$
\begin{aligned}
& \mathcal{L}_{\text {surv }}^{A}=\mathcal{L}_{U}^{A}+\alpha^{A} \mathcal{L}_{C}^{A}+\mathcal{L}_{\text {prior }}, \quad \text { where } \\
& \mathcal{L}_{U}^{A}=\sum_{a} \sum_{i=1}^{\left|\mathcal{D}_{U}\right|} \sigma_{K}\left(\ln f^{a}\left(y_{i}^{a} \mid \beta_{k_{i}}^{a}, \eta_{k_{i}}^{a}\right)\right), \\
& \mathcal{L}_{C}^{A}=\sum_{a} \sum_{i=1}^{\left|\mathcal{D}_{C}\right|} \sigma_{K}\left(\ln S^{a}\left(y_{i}^{a} \mid \beta_{k_{i}}^{a}, \eta_{k_{i}}^{a}\right)\right),
\end{aligned}
$$

where $a \in\{0,1\}, \alpha^{A}$ is a scalar hyperparameter as defined in Sec. III-A.

Proof: The log-likelihood for $\mathcal{D}$ is be given by

$$
\begin{aligned}
\ln P\left(\mathcal{D} \mid \Omega^{A}\right) & =\sum_{i=1}^{\left|\mathcal{D}_{U}\right|} \ln \left(P\left(Y_{T}=y_{T, i} \mid \mathbf{X}=\mathbf{x}_{i}, \Omega^{A}\right)\right. \\
& +\sum_{j=1}^{\left|\mathcal{D}_{C}\right|} \ln P\left(Y_{T}>y_{C, j} \mid \mathbf{X}=\mathbf{x}_{j}, \Omega^{A}\right),
\end{aligned}
$$

where $\Omega^{A}$ represents the set of parameters, $\mathcal{D}_{U} \subset \mathcal{D}$ consists of instances $\mathbf{x}_{i}$ that are not censored, $\mathcal{D}_{C} \subset \mathcal{D}$ consists of instances that are censored, and $\mathcal{D}_{U} \cup \mathcal{D}_{C}=\mathcal{D}$. The first term on the right hand side above can be expressed in terms of the treatment provided as

$$
\begin{aligned}
& \sum_{i=1}^{\left|\mathcal{D}_{U}\right|} \ln P\left(Y_{T}=y_{T, i} \mid \mathbf{X}=\mathbf{x}_{i}, \Omega^{A}\right) \\
& \quad=\sum_{i=1}^{\left|\mathcal{D}_{U}\right|: a=1} \ln \left(P\left(Y_{T}^{1}=y_{T, i}^{1} \mid \mathbf{X}=\mathbf{x}_{i}, \Omega^{A}\right)\right. \\
& \quad+\sum_{i=1}^{\left|\mathcal{D}_{U}\right|: a=0} \ln P\left(Y_{T}^{0}=y_{T, i}^{0} \mid \mathbf{X}=\mathbf{x}_{i}, \Omega^{A}\right) \\
& \quad=\sum_{i=1}^{\left|\mathcal{D}_{U}\right|}\left(\operatorname { l n } \left(P\left(Y_{T}=y_{T, i}^{1} \mid \mathbf{X}=\mathbf{x}_{i}, a=1, \Omega^{A}\right)\right.\right. \\
& \left.\quad+\ln P\left(Y_{T}=y_{T, i}^{0} \mid \mathbf{X}=\mathbf{x}_{i}, a=0, \Omega^{A}\right)\right)
\end{aligned}
$$

where we obtained the last equation above using the ignorability assumption stated in Sec. III-C. Using mixture distribution membership $Z_{i}^{a}$ which takes one of the $K$ values per cluster 


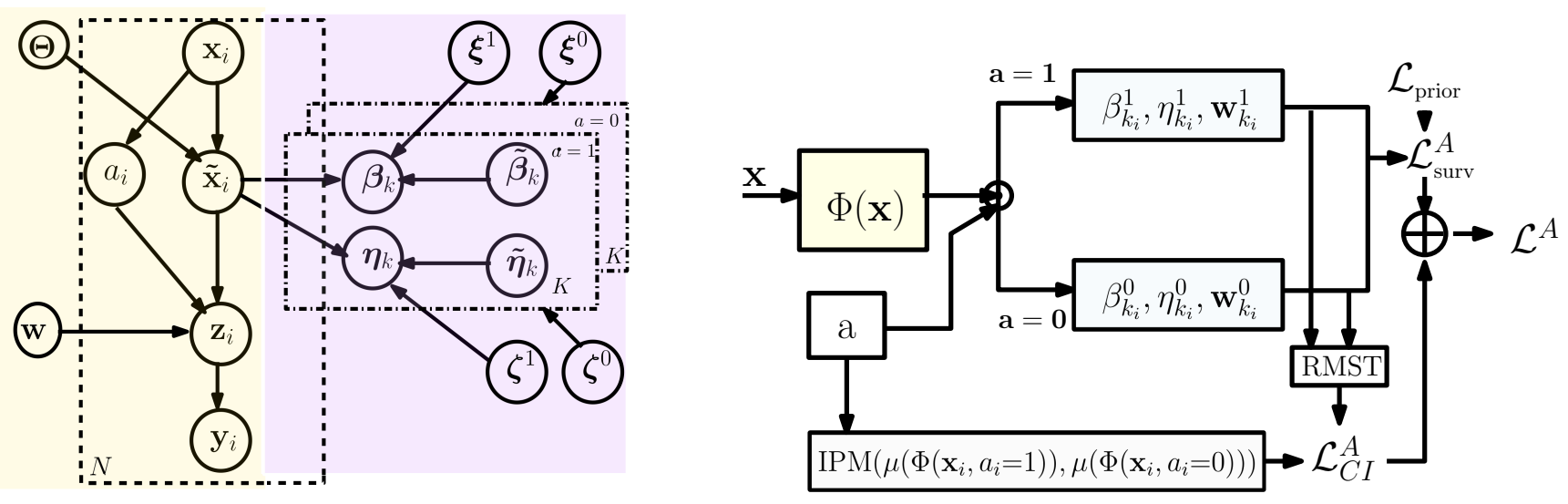

Fig. 2. BN for the SurvCI model is given on the left, where region shaded in light-yellow represents the causal relationships and encapsulates $\mathbf{\Phi}(\cdot)$, while the region in violet represent the parameters for survival time. The proposed SurvCI architecture is depicted on the right. This architecture consists of representation layer $\boldsymbol{\Phi}(\cdot)$, RMST, IPM module and the hypothesis layers that predict the parameters of the $\boldsymbol{K}$ mixture distributions.

represented by $k_{i}=1, \ldots, K$ as the latent variable, we rewrite the above expression as

$\sum_{i=1}^{\left|\mathcal{D}_{U}\right|} \ln P\left(Y_{T}=y_{T, i} \mid \mathbf{X}=\mathbf{x}_{i}, \Omega^{A}\right)=\sum_{i=1}^{\left|\mathcal{D}_{U}\right|}$

$\left(\ln \sum_{k=1}^{K} P\left(Y_{T}=y_{T, i}^{1} \mid Z_{i}^{1}, \beta_{k}^{1}, \eta_{k}^{1}\right) P\left(Z_{i}^{1} \mid \mathbf{X}=\mathbf{x}_{i}, a=1, \Omega^{A}\right)\right.$

$\left.+\ln \sum_{k=1}^{K} P\left(Y_{T}=y_{T, i}^{0} \mid Z_{i}^{0}, \beta_{k}^{0}, \eta_{k}^{0}\right) p\left(Z_{i}^{0} \mid \mathbf{X}=\mathbf{x}_{i}, a=0, \Omega^{A}\right)\right)$,

where $\beta_{k_{i}}^{a}$ and $\eta_{k_{i}}^{a}$ are treatment and cluster-specific parameters. This latent variable formulation results in the outcome being dependent on the latent variables, while latent variable depends on the input covariates and the treatment, as depicted in Fig. 2. Applying Jensen's inequality, we obtain the ELBO formulation in the presence of two treatments as follows:

$$
\begin{aligned}
& \sum_{i=1}^{\left|\mathcal{D}_{U}\right|} \ln P\left(Y_{T}=y_{T, i} \mid \mathbf{X}=\mathbf{x}_{i}, \Omega^{A}\right) \\
& \geq \sum_{i=1}^{\left|\mathcal{D}_{U}\right|}\left[\mathbb{E}_{Z_{i}^{1}}\left(\ln P\left(Y_{T}=y_{T, i}^{1} \mid Z_{i}^{1}, \beta_{k}^{1}, \eta_{k}^{1}\right)\right)\right. \\
& \left.+\mathbb{E}_{Z_{i}^{0}}\left(\ln P\left(Y_{T}=y_{T, i}^{0} \mid Z_{i}^{0}, \beta_{k}^{0}, \eta_{k}^{0}\right)\right)\right] .
\end{aligned}
$$

The above expression can be written in terms of the failure density and the survival function as

$$
\begin{aligned}
& \sum_{i=1}^{\left|\mathcal{D}_{U}\right|} \ln P\left(Y_{T}=y_{T, i} \mid \mathbf{X}=\mathbf{x}_{i}, \Omega^{A}\right) \\
& \geq \sum_{i=1}^{\left|\mathcal{D}_{U}\right|}\left[\sigma_{K}\left(\ln f^{1}\left(y_{T, i}^{1} \mid \beta_{k_{i}}^{1}, \eta_{k_{i}}^{1}\right)\right)+\sigma_{K}\left(\ln f^{0}\left(y_{T, i}^{0} \mid \beta_{k_{i}}^{0}, \eta_{k_{i}}^{0}\right)\right)\right] \\
& =\mathcal{L}_{U}^{A},
\end{aligned}
$$

where $f^{a}\left(y_{i}^{a} \mid \beta_{k_{i}}^{a}, \eta_{k_{i}}^{a}\right)$ represents the failure density w.r.t. the treatment $a$. Similarly, for censored data $\mathcal{D}_{C}$, the second term on the right hand side of (14) can be simplified as

$$
\begin{aligned}
& \sum_{i=1}^{\left|\mathcal{D}_{C}\right|} \ln P\left(Y_{T}>y_{C, i} \mid \mathbf{X}=\mathbf{x}_{i}, \Omega^{A}\right) \\
& \geq \sum_{i=1}^{\left|\mathcal{D}_{C}\right|} \sigma_{K}\left(\ln S^{1}\left(y_{C, i}^{1} \mid \beta_{k_{i}}^{1}, \eta_{k_{i}}^{1}\right)\right)+\sigma_{K}\left(\ln S^{0}\left(y_{C, i}^{0} \mid \beta_{k_{i}}^{0}, \eta_{k_{i}}^{0}\right)\right) \\
& =\mathcal{L}_{C}^{A} .
\end{aligned}
$$

Inspired by [12], we utilize a scalar hyperparameter $\alpha^{A}$ and consider the weighted (18) and (19) and substitute into (14).

From the above expression, it is evident that learning $\Omega^{A}=$ $\left\{\Theta, \mathbf{w}_{i}^{a}, \beta_{k}^{a}, \eta_{k}^{a}\right\}$ for all $i$ is specific to $a$, i.e., learning these parameters entail training samples that receive treatment $a$. In summary, theorem 1 showed that extending the DSM based cost function in the context of binary treatments leads to two independent entities in the cost function, one per treatment, since the terms in ELBO separate out with respect to the two treatments. In terms of a neural network architecture, one can naturally envisage a DNN with two branches, one for each treatment, as depicted in Fig. 2. The input to the SurvCI model are the covariates $\mathbf{x}_{i}$, which are transformed to $\tilde{\mathbf{x}}_{i}$ by the representation layer $\Phi(\cdot)$, in order to mitigate the effect of selection bias in observational data. Effectively, $\psi(\cdot)$ in the DSM architecture (Sec. III-A) is replaced by the representation $\Phi(\cdot)$. The representation layer is trained using the $\operatorname{IPM}(\cdot)$, as detailed on Sec. III-C. Based on the treatment indicator $a$, the model branches into hypothesis layers, one for treated $(a=1)$ and the other for control $(a=0)$, as shown in Fig. 2 (on the right). The cost function $\mathcal{L}_{C I}$ includes the squared error based loss, $L(\cdot, \cdot)$, which is computed as

$$
\begin{aligned}
L\left(h\left(\Phi\left(\mathbf{x}_{i}, a_{i}\right)\right), y_{i}^{a}\right) & =\left\|h\left(\Phi\left(\mathbf{x}_{i}, a_{i}\right)\right)-y_{i}^{a}\right\|_{2}^{2} \\
& =\left\|\int_{0}^{T^{*}} \hat{S}^{a}\left(y_{T, i}^{a} \mid \mathbf{x}_{i}\right) d y_{T}-y_{T, i}^{a}\right\|_{2}^{2} .
\end{aligned}
$$

Note that each branch of the hypothesis layer in Fig. 2 computes the updates of the parameters $\left\{\beta_{k}^{a}, \eta_{k}^{a}, \mathbf{w}_{k_{i}}^{a}\right\}$ using which, the distribution of per-individual survival time is determined. This allows us to compute the right hand side of (36). Since RMST is defined for both event and censored observations, $\mathcal{L}_{C I}$ incorporates information from all observations. The final loss function is given by $\mathcal{L}^{A}$ given as

$$
\mathcal{L}^{A}=\rho^{A} \mathcal{L}_{\text {surv }}^{A}+\mathcal{L}_{C I},
$$

where the expression for $\mathcal{L}_{\text {surv }}^{A}$ is as given in (13) and $\rho^{A}$ is a hyperparameter. During training, the model determines parameters $\left\{\beta_{k}^{a}, \eta_{k}^{a}, \mathbf{w}^{a}, \xi, \zeta\right\}_{k=1}^{K}$ and $a \in\{0,1\}$. The final survival distribution per instance is obtained by mixing over these $K$ distributions using the learnt parameters. One question that remains is regarding how to obtain the predicted outcome $\hat{y}_{T, i}^{0}$ or $\hat{y}_{T, i}^{1}$. Mathematically and from (11), it is clear that the outcome survival time under each treatment can be obtained 
as follows:

$$
\hat{y}_{T, i}^{a}=\mathbb{E}\left[y_{T, i} \mid \mathbf{x}_{i}, a\right]=\int_{0}^{T^{*}} \hat{S}^{a}\left(y_{T}^{a} \mid \mathbf{x}_{i}\right) d y_{T},
$$

i.e., the survival time is the area under the predicted survival function for the given treatment $a$. In SurvCI, after the training phase, we obtain the estimates of the parameters of the mixture densities namely $\left\{\eta_{k}^{a}, \beta_{k}^{a}, \mathbf{w}_{k}\right\}$ for all $k$ and $a$. Since the form of the primitive forms of the distributions are also known, we have the survival density per individual. We empirically evaluate the right hand side of (22) and obtain $y_{T}^{a}$.

\section{SURVCI-InFo FOR INFORMATIVE CEnSORED DATA}

In this section, we formulate the generative modeling based ELBO framework to account for informative censoring by considering user-dependent time-to-censoring as an outcome [20], [36] alongside time-to-event. Similar to time-to-event $y_{T}$, we model the conditional distribution of time-to-censoring, $y_{C} \sim P\left(Y_{C} \mid \mathbf{X}=\mathbf{x}\right)$ as a mixture over $K$ well-defined, parametric primitive distributions.

\section{A. ELBO for SA with Informative Censoring}

The ELBO formulation in the context of informative censoring is interesting since it pans out as an interplay between event and censoring times, $Y_{T}$ and $Y_{C}$, respectively. As in [20], we assume that $\left(Y_{C} \Perp Y_{T} \mid \mathbf{X}\right)$, i.e., a conditionally independent informative censoring model. For clarity purposes, we first extend the ELBO framework [12] to include informative censoring, and extend it further to include binary treatments. In the presence of random censoring, only time-to-event is modeled and the censored outcomes are accounted for in the loss function via likelihood. However, if the censoring depends upon the covariates, it is essential to mathematically model time-to-censoring, and learn the function that characterizes the per-instance failure density. Although predicting timeto-censoring also allows us to predict the binary censoring indicator, $\delta$, for unseen samples, we do not use this functionality in this work as ground truth $\delta$ is available. In the following formulation, we derive the ELBO loss function for the informative censoring case, where we model both, $Y_{T}$ and $Y_{C}$.

Proposition 2: The overall ELBO-based loss function for the survival dataset $\mathcal{D}$ in the presence of informative censoring is given by

$$
\begin{aligned}
& \mathcal{L}_{\text {surv }}^{I}=\mathcal{L}_{U}^{I}+\alpha^{I} \mathcal{L}_{C}^{I}+\mathcal{L}_{\text {prior }}, \quad \text { where } \\
\mathcal{L}_{U}^{I}= & \sigma_{K}\left(\ln f_{T}\left(y_{T, i} \mid \beta_{T, k_{i}}, \eta_{T, k_{i}}\right)\right) \\
& +\sigma_{K}\left(\ln S_{T}\left(y_{T, i} \mid \beta_{C, k_{i}}, \eta_{C, k_{i}}\right)\right) \\
\mathcal{L}_{C}^{I}= & \sigma_{K}\left(\ln S_{C}\left(y_{C, i} \mid \beta_{T, k_{i}}, \eta_{T, k_{i}}\right)\right) \\
& +\sigma_{K}\left(\ln f_{C}\left(y_{C, i} \mid \beta_{C, k_{i}}, \eta_{C, k_{i}}\right)\right)
\end{aligned}
$$

where $\alpha^{I}$ is hyperparameter as defined in Sec. III-A, $f_{T}(\cdot)$ and $S_{T}(\cdot)$ represent the failure and survival density for time to event, $f_{C}(\cdot)$ and $S_{C}(\cdot)$ represent the failure and survival density for censoring time and $\mathcal{L}_{\text {prior }}$ is as given in (5).
Proof: Considering both $Y_{T}$ and $Y_{C}$, the log-likelihood for event data $\mathcal{D}_{U}$ is given by

$$
\begin{aligned}
& \ln P\left(\mathcal{D}_{U} \mid \Omega^{I}\right) \\
& =\sum_{i=1}^{\left|\mathcal{D}_{U}\right|} \ln \left(P\left(Y_{T}=y_{T, i}, Y_{C}>y_{T, i} \mid \mathbf{X}=\mathbf{x}_{i}, \Omega^{I}\right),\right.
\end{aligned}
$$

where the probabilistic event $\left(Y_{T}=y_{T, i}, Y_{C}>y_{T, i}\right)$ encompasses the cases where event has occurred at $y_{T, i}$, and hence, as a consequence, censoring occurs is after $y_{T, i}$. Using $Y_{C} \Perp Y_{T} \mid \mathbf{X}$, the above expression can be written as

$$
\begin{aligned}
& \ln P\left(\mathcal{D}_{U} \mid \Omega^{I}\right)=\sum_{i=1}^{\left|\mathcal{D}_{U}\right|} \ln \left(P\left(Y_{T}=y_{T, i} \mid \mathbf{X}=\mathbf{x}_{i}, \Omega^{I}\right)\right. \\
& \quad+\ln P\left(Y_{C}>y_{T, i} \mid \mathbf{X}=\mathbf{x}_{i}, \Omega^{I}\right) .
\end{aligned}
$$

Introducing the latent variables $Z_{T}$ and $Z_{C}$ and applying Jensen's inequality, we obtain the following:

$$
\begin{aligned}
& \ln P\left(\mathcal{D}_{U} \mid \Omega^{I}\right) \\
& \geq \sum_{i=1}^{\left|\mathcal{D}_{U}\right|} \mathbb{E}_{Z_{T, i}}\left[\ln P\left(Y_{T}=y_{T, i} \mid Z_{T, i}, \beta_{T, k}, \eta_{T, k}\right)\right. \\
& \left.+\ln P\left(Y_{C}>y_{T, i} \mid Z_{C, i}, \beta_{C, k}, \eta_{C, k}\right)\right] \geq \\
& \sigma_{K}\left(\ln f_{T}\left(y_{T, i} \mid \beta_{T, k_{i}}, \eta_{T, k_{i}}\right)\right)+\sigma_{K}\left(\ln S_{T}\left(y_{T, i} \mid \beta_{C, k_{i}}, \eta_{C, k_{i}}\right)\right) \\
& =\mathcal{L}_{U}^{I} .
\end{aligned}
$$

Similarly for censored data, the ELBO loss is as follows:

$$
\begin{aligned}
& \ln P\left(\mathcal{D}_{C} \mid \Omega^{I}\right)= \\
& \sum_{i=1}^{\left|\mathcal{D}_{C}\right|} \ln \left(P\left(Y_{T}>y_{C, i}, Y_{C}=y_{C, i} \mid \mathbf{X}=\mathbf{x}_{i}, \Omega^{I}\right),\right.
\end{aligned}
$$

where the probabilistic event $\left(Y_{T}>y_{C, i}, Y_{C}=y_{C, i}\right)$ consists of those where event has not occurred until $y_{C, i}$, i.e., $Y_{T}>$ $y_{C, i}$, and hence, the time at which censoring occurs, $Y_{C}=$ $y_{C, i}$. Following the steps as earlier, we obtain the following:

$$
\begin{aligned}
& \ln P\left(\mathcal{D}_{C} \mid \Omega^{I}\right) \geq \sum_{i=1}^{\left|\mathcal{D}_{C}\right|} \sigma_{K}\left(\ln S_{C}\left(y_{C, i} \mid \beta_{T, k_{i}}, \eta_{T, k_{i}}\right)\right) \\
& \quad+\sigma_{K}\left(\ln f_{C}\left(y_{C, i} \mid \beta_{C, k_{i}}, \eta_{C, k_{i}}\right)\right)=\mathcal{L}_{C}^{I} .
\end{aligned}
$$

Including the prior term as earlier, the overall cost function for survival analysis is given by the sum of (26) and (28), i.e.,

$$
\mathcal{L}_{\text {surv }}^{I}=\mathcal{L}_{U}^{I}+\alpha^{I} \mathcal{L}_{C}^{I}+\mathcal{L}_{\text {prior }},
$$

where $\alpha^{I}$ is a hyperparameter as defined earlier. Note that $\Omega^{I}$ represents the set of parameters given by $\Omega^{I}=$ $\left\{\Theta, \mathbf{w}_{T}, \mathbf{w}_{C}, \beta_{T, k}, \beta_{C, k}, \eta_{T, k}, \eta_{C, k}\right\}$ for $a \in\{0,1\}$ and $\forall k$.

In the following theorem, we extend the above obtained informative-censoring based ELBO loss function to include binary treatments.

Theorem 2: The ELBO-based loss function for the survival dataset $\mathcal{D}$ in the presence of informative censoring and binary treatments is given by

$$
\begin{aligned}
\mathcal{L}_{\text {surv }}^{I A} & =\mathcal{L}_{U}^{I A}+\alpha^{I A} \mathcal{L}_{C}^{I A}+\mathcal{L}_{\text {prior }}, \\
\mathcal{L}_{U}^{I A} & =\sum_{a}\left(\sigma_{K}\left(\ln f_{T}^{a}\left(y_{T, i}^{a} \mid \beta_{T, k_{i}}^{a}, \eta_{T, k_{i}}^{a}\right)\right)\right. \\
& \left.+\sigma_{K}\left(\ln S_{T}^{a}\left(y_{T, i}^{a} \mid \beta_{C, k_{i}}^{a}, \eta_{C, k_{i}}^{a}\right)\right)\right) \\
\mathcal{L}_{C}^{I A} & =\sum_{a}\left(\sigma_{K}\left(\ln S_{C}^{a}\left(y_{C, i}^{a} \mid \beta_{T, k_{i}}^{a}, \eta_{T, k_{i}}^{a}\right)\right)\right. \\
& +\sigma_{K}\left(\ln f_{C}^{a}\left(y_{C, i}^{a} \mid \beta_{C, k_{i}}^{a}, \eta_{C, k_{i}}^{a}\right)\right),
\end{aligned}
$$

where $a \in\{0,1\}, \alpha^{I A}$ is hyperparameter as defined in Sec. III$\mathrm{A}, f_{T}^{a}(\cdot)$ and $S_{T}^{a}(\cdot)$ represent the failure and survival density, 


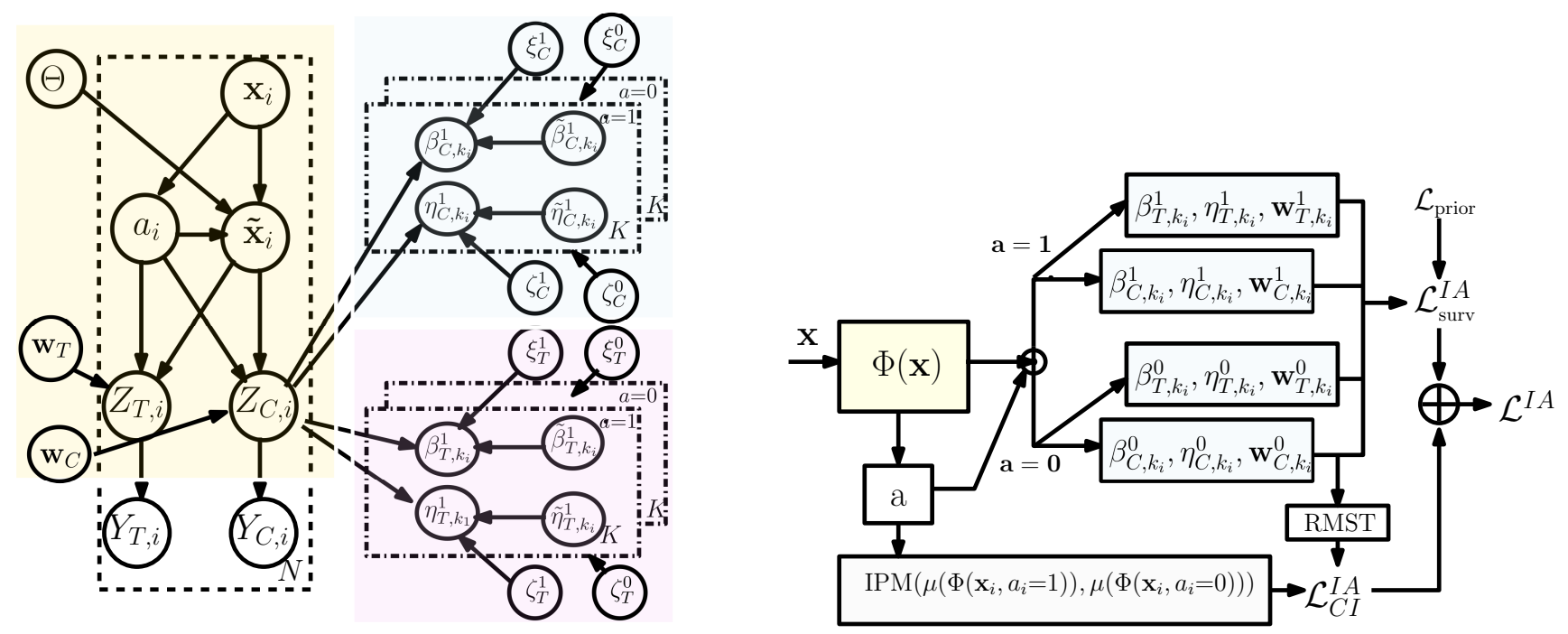

Fig. 3. On the left we depict the BN for SurvCI-Info. Here, the region shaded in yellow represents the representation $\Phi(\cdot)$ layer and associated parameterization, light-pink and light-blue regions represent the parameters for time to event and time to censoring, respectively. Dashed-style boxes denote the plate notation. On the right, we depict the architecture of the proposed SurvCI-Info model shows representation $\mathbf{\Phi}(\mathbf{x})$, RMST, IPM module, and the SA-based hypotheses layers that learns the parameters of the components of the $\boldsymbol{K}$ mixture distributions.

respectively, for time to event and treatment $a, f_{C}^{a}(\cdot)$ and $S_{C}^{a}(\cdot)$ represent the failure and survival density, respectively, for censoring time, for a given treatment $a$, and $\mathcal{L}_{\text {prior }}$ is as given in (5).

Proof: The log-likelihood for event data in the presence of binary treatments is given by:

$$
\begin{aligned}
& \ln P\left(\mathcal{D}_{U} \mid \Omega^{I A}\right)= \\
& \sum_{i=1}^{\left|\mathcal{D}_{U}\right|: a=1} \ln \left(P\left(Y_{T}^{1}=y_{T, i}^{1}, Y_{C}^{1}>y_{T, i}^{1} \mid \mathbf{x}=\mathbf{x}_{i}, \Omega^{I A}\right)\right. \\
& \left.+\sum_{i=1}^{\left|\mathcal{D}_{U}\right|: a=0} \ln P\left(Y_{T}^{0}=y_{T, i}^{0}, Y_{C}^{0}>y_{T, i}^{0} \mid \mathbf{x}=\mathbf{x}_{i}, \Omega^{I A}\right)\right),
\end{aligned}
$$

where $\Omega^{I A}$ represents the set of parameters to be learnt in the context of SurvCI-Info model. In order to simplify the RHS above, we apply conditional ignorability condition and convert the treatment specific outcome distribution into conditional densities (conditioned on the treatment). Further, we use $Y_{C} \Perp Y_{T} \mid \mathbf{X}$ and apply the Jensen's inequality to obtain:

$$
\begin{aligned}
& \ln P\left(\mathcal{D}_{U} \mid \Omega^{I A}\right) \\
& \geq \sum_{i=1}^{\left|\mathcal{D}_{U}\right|} \mathbb{E}_{Z_{T, i}^{1}}\left[\ln P\left(Y_{T}^{1}=y_{T, i}^{1} \mid Z_{T, i}^{1}, \beta_{T, k}^{1}, \eta_{T, k}^{1}\right)\right] \\
& +\mathbb{E}_{Z_{C}^{1}}\left[\ln P\left(Y_{C}^{1}>y_{T, i}^{1} \mid Z_{C, i}^{1}, \beta_{C, k}^{1}, \eta_{C, k}^{1}\right)\right] \\
& +\mathbb{E}_{Z_{T}^{0}}\left[\ln P\left(Y_{T}^{0}=y_{T, i}^{0} \mid Z_{T, i}^{0}, \beta_{T, k}^{0}, \eta_{T, k}^{0}\right)\right] \\
& +\mathbb{E}_{Z_{C}^{0}}^{0}\left[\ln P\left(Y_{C}^{0}>y_{T, i}^{0} \mid Z_{C, i}^{0}, \beta_{C, k}^{0}, \eta_{C, k}^{0}\right)\right] \\
& =\sigma_{K}\left(\ln f_{T}^{1}\left(y_{T, i}^{1} \mid \beta_{T, k_{i}}^{1}, \eta_{T, k_{i}}^{1}\right)\right)+\sigma_{K}\left(\ln S_{T}^{1}\left(y_{T, i}^{1} \mid \beta_{C, k_{i}}^{1}, \eta_{C, k_{i}}^{1}\right)\right) \\
& +\sigma_{K}\left(\ln f_{T}^{0}\left(y_{T, i}^{0} \mid \beta_{T, k_{i}}^{0}, \eta_{T, k_{i}}^{0}\right)\right)+\sigma_{K}\left(\ln S_{T}^{0}\left(y_{T, i}^{0} \mid \beta_{C, k_{i}}^{0}, \eta_{C, k_{i}}^{0}\right)\right) \\
& =\mathcal{L}_{U}^{I A} .
\end{aligned}
$$

Similarly, for censored data $\mathcal{D}_{C}$, we obtain the joint ELBO loss in the presence of two treatments as

$$
\begin{aligned}
& \ln P\left(\mathcal{D}_{C} \mid \Omega^{I A}\right) \geq \sum_{i=1}^{\left|\mathcal{D}_{C}\right|} \sigma_{K}\left(\ln S_{C}^{1}\left(y_{C, i}^{1} \mid \beta_{T, k_{i}}^{1}, \eta_{T, k_{i}}^{1}\right)\right) \\
& +\sigma_{K}\left(\ln f_{C}^{1}\left(y_{C, i}^{1} \mid \beta_{C, k_{i}}^{1}, \eta_{C, k_{i}}^{1}\right)\right)+\sigma_{K}\left(\ln S_{C}^{0}\left(y_{C, i}^{0} \mid \beta_{T, k_{i}}^{0}, \eta_{T, k_{i}}^{0}\right)\right) \\
& +\sigma_{K}\left(\ln f_{C}^{0}\left(y_{C, i}^{0} \mid \beta_{C, k_{i}}^{0}, \eta_{C, k_{i}}^{0}\right)\right)=\mathcal{L}_{C}^{I A} .
\end{aligned}
$$

Combining the prior term given in (5), the overall loss function is given by the sum of (32) and (33), i.e.,

$$
\mathcal{L}_{\text {surv }}^{I A}=\mathcal{L}_{U}^{I A}+\alpha^{I A} \mathcal{L}_{C}^{I A}+\mathcal{L}_{\text {prior }},
$$

where $\alpha^{I A}$ is the hyperparameter as defined earlier.

From the above expression, it is evident that in training the informative SurvCI-Info model involves learning of parameters $\Omega^{I A}=\left\{\Theta, \mathbf{w}_{T}, \mathbf{w}_{C}, \beta_{T, k}^{a}, \beta_{C, k}^{a}, \eta_{T, k}^{a}, \eta_{C, k}^{a}\right\}$ for $a \in\{0,1\}$ and all $k$. In summary, theorem 2 showed that extending the SurvCI based cost function in the presence of informative censoring leads to four entities in the cost function, one per treatment for both, time-to-event and timeto-censoring. Evidently, this is achieved since the terms in ELBO separate out with respect to the treatments. As depicted in Fig. 3, the input to the SurvCI-Info model are the covariates $\mathbf{x}_{i}$, which are transformed to $\tilde{\mathbf{x}}_{i}$ by the representation layer $\Phi(\cdot)$, in order to mitigate the effect of selection bias in observational data and the representation layer is trained using the $\operatorname{IPM}(\cdot)$, which is a part of (8). Based on the treatment indicator $a$, the model branches into hypothesis layers, two for treated (one each for $T$ and $C)(a=1)$ and the other two for control $(a=0)$, as shown in Fig. 3 (on the right). The final loss function is given by $\mathcal{L}^{I A}$ given as

$$
\mathcal{L}^{I A}=\rho^{I A} \mathcal{L}_{\text {surv }}^{I A}+\mathcal{L}_{C I},
$$

where $\rho^{I A}$ is a hyperparameter, $\mathcal{L}_{\text {surv }}$ is as given in prop. 2, $\mathcal{L}_{C I}$ is computed using (36) and includes the squared error based loss and $L(\cdot, \cdot)$, which is computed as

$$
\begin{aligned}
& L\left(h\left(\Phi\left(\mathbf{x}_{i}, a_{i}\right)\right), y_{i}^{a}\right)=\left\|h\left(\Phi\left(\mathbf{x}_{i}, a_{i}\right)\right)-y_{i}^{a}\right\|_{2}^{2} \\
& =\left\|\int_{0}^{T^{*}} \hat{S}_{T}^{a}\left(y_{T, i}^{a} \mid \mathbf{x}\right) d y_{T}-y_{T, i}^{a}\right\|_{2}^{2} \\
& \quad+\left\|\int_{0}^{C^{*}} \hat{S}_{C}^{a}\left(y_{T, i}^{a} \mid \mathbf{x}\right) d y_{C}-y_{C, i}^{a}\right\|_{2}^{2} .
\end{aligned}
$$

In Fig. 3, the boxes highlighted in blue and pink are the parameters for $a=1$ and $a=0$, respectively. The blocks in the 


\begin{tabular}{|c|c|c|c|c|c|}
\hline Dataset & $\mathrm{D}$ & $\left|\mathcal{D}_{C}\right|(\%)$ & $\left|\mathcal{D}_{U}\right|(\%)$ & $N_{1}$ & $N_{0}$ \\
\hline Synthetic & 12 & 27.9 & 23.08 & 1154 & 3846 \\
\hline Synthetic-Info & 12 & 38.9 & 24.04 & 1202 & 3798 \\
\hline ACTG-Semi & 23 & 51.06 & 55.9 & 1196 & 943 \\
\hline ACTGInfo-Semi & 23 & 43.94 & 44.44 & 1189 & 950 \\
\hline Metabric & 25 & 42.28 & 59.24 & 1173 & 807 \\
\hline
\end{tabular}

TABLE II

Descriptive Statistics of the Datasets.

hypothesis layer estimate parameters for time-to-censoring and the dashed-style is the plate notation denoting i.i.d data. The final individual survival distribution for the event and censoring time, $Y_{T}$ and $Y_{C}$ is a weighted average over corresponding $K$ distributions, for each of the treatments, i.e., we learn $4 K$ parameters in total. Note that if we eliminate $Z_{C}^{a}$ (shaded in blue) and $Y_{C}^{a}$, and the corresponding parameters, we obtain the BN for the SurvCI model. Similar to (22), the predicted survival time is obtained using the learnt parameters. The predicted outcome $\hat{y}_{T, i}^{a}$ and $\hat{y}_{C, i}^{a}$ is obtained by obtaining the area under the curve of the corresponding survival densities for $Y_{T}$ and $Y_{C}$, respectively.

\section{EXPERIMENTS AND REsults}

In this section, we demonstrate the efficacy of the proposed SurvCI and SurvCI-Info techniques and compare its performance with several state-of-the-art benchmarks. We first describe the data sets employed in the experiments, followed by the baselines, metrics, results and discussions. ${ }^{2}$

\section{A. Datasets}

The datasets used to illustrate the results are as follows:

- Synthetic Non-informative Dataset: We use a synthetic dataset with three stochastic processes (one for the covariates, the other two for event and censoring times, respectively) [12], [19]. This dataset consists of 12 covariates, grouped into $\mathbf{x}_{1}^{(i)}, \mathbf{x}_{2}^{(i)}, \mathbf{x}_{3}^{(i)} \sim \mathcal{N}(0,1)$. Further, $Y_{T, i}^{(0)} \sim \exp \left(\left(\gamma_{3}^{T} \mathbf{x}_{3}^{(i)}\right)^{2}+\gamma_{1}^{T} \mathbf{x}_{1}^{(i)}\right)$ and $Y_{T, i}^{(1)} \sim$ $\exp \left(\left(\gamma_{3}^{T} \mathbf{x}_{3}^{(i)}\right)^{2}+\gamma_{2}^{T} \mathbf{x}_{2}^{(i)}\right)$. We assume $\gamma_{1}=\gamma_{2}=\gamma_{3}$ to be in $\mathbb{R}^{4}$ and each entry of these vectors is 10 . To introduce selection bias, we force the treatment assignment to be depend on the covariates,i.e., if $\mathbf{x}_{i} \in[-1,1]$, then $t_{i}=1$, and $t_{i}=0$ otherwise, for all $i$. Further, we generate time to censoring as $\log \left(Y_{C, i}^{t}\right) \sim \mathcal{N}\left(\mu_{c}, \sigma_{c}^{2}\right)$ and $Y_{T, i}^{t}=$ $\min \left(Y_{T, i}^{t}, Y_{C, i}^{t}\right), \quad \forall i, a$. Here, $Y_{C, i}^{t}$ denotes the censoring time for treatment $t$. We set $\delta=1$ if $Y_{T, i}^{t}<Y_{C, i}^{t}$, and $\delta=0$ otherwise. Further, we the hyperparameters $\mu_{c}=1200$ and $\sigma_{c}^{2}=2500$.

- Synthetic Informative Dataset: Synthetic informative dataset consists of three stochastic processes with 12 covariates, grouped into $\mathbf{x}_{1}^{(i)}, \mathbf{x}_{2}^{(i)}, \mathbf{x}_{3}^{(i)} \sim \mathcal{N}(1,1)$. Further, $Y_{T, i}^{(0)} \sim \exp \left(\left(\gamma_{4}^{T} \mathbf{x}_{3}^{(i)}\right)^{2}+\gamma_{4}^{T} \mathbf{x}_{1}^{(i)}\right)$ and $Y_{T, i}^{(1)} \sim$ $\exp \left(\left(\gamma_{4}^{T} \mathbf{x}_{3}^{(i)}\right)^{2}+\gamma_{4}^{T} \mathbf{x}_{2}^{(i)}\right)$, where $\gamma_{4} \in \mathbb{R}^{4}$ and each entry is 5 . Further, instance-dependent time-to-censoring is generated as $Y_{C, i}^{(0)} \sim \exp \left(\left(\gamma_{5}^{T} \mathbf{x}_{3}^{(i)}\right)^{2}+\gamma_{5}^{T} \mathbf{x}_{1}^{(i)}\right)$ and

${ }^{2}$ The code is available at github.com/muskang $48 /$ SurvCI.
$Y_{C, i}^{(1)} \sim \exp \left(\left(\gamma_{5}^{T} \mathbf{x}_{3}^{(i)}\right)^{2}+\gamma_{5}^{T} \mathbf{x}_{2}^{(i)}\right)$, where $\gamma_{5} \in \mathbb{R}^{4}$ and each entry is 80 . We generate the final outcome as $\min \left(Y_{T, i}^{t}, Y_{C, i}^{t}\right), \forall i, a$ and corresponding $\delta_{i}$ is generated. The other details of generation remain similar to the randomly-censored synthetic dataset.

- ACTG-Semi synthetic dataset: The AIDS Clinical Trials Group (ACTG) is an RCT study [42]. Generation of the semi-synthetic dataset based on covariates from ACTG is as described in [33]. Generation of the semi-synthetic dataset based on covariates from ACTG is as described in [33]. The time-to-censoring for the non-informative censoring scenario is generated as $\log \left(Y_{C, i}\right) \sim \mathcal{N}\left(\mu_{c}, \sigma_{c}^{2}\right)$. Further, for informative censoring case, time-to-censoring is generated as $\log \left(Y_{C, i}^{t}\right) \sim \frac{1}{\alpha_{C}^{a}} \log (1-) \frac{\alpha_{c}^{a} \log U}{\lambda_{c}^{a} \exp \left(\mathbf{x}^{T} \beta_{a}\right)}$, where $U \sim \operatorname{Unif}(0,1), \alpha_{c}^{a}=5 e^{-3}$ and $\lambda_{c}^{a}=8.8 e^{-4}$.

- Real-world Metabric Dataset: The Molecular Taxonomy of Breast Cancer International Consortium (METABRIC) dataset is a SA dataset that consists of 1,981 treated breast cancer (BC) patients. It consists of gene expression profiles and clinical features of patients, with event being death. This dataset consists of four non-exclusive treatments namely radiotherapy (RT), chemotherapy (CT), breast surgery and hormone therapy.

\begin{tabular}{|c|c|c|c|c|}
\hline Method & $\mathrm{C}_{I}^{(a=0, a=1)}$ & $\epsilon_{A T E}$ & $\sqrt{\epsilon_{P E H E}}$ & $\mathrm{HR}(a)$ \\
\hline DSM & $(\mathbf{0 . 7 7 9 , 0 . 7 6 2 )}$ & - & - & 1.07 \\
\hline CRFNet & $(0.646,0.676)$ & 12.51 & 249.24 & - \\
\hline CSA & $(0.771,0.751)$ & 33.98 & 1261.63 & 1.73 \\
\hline$\kappa$-SurvCI & $(0.751,0.757)$ & 2.10 & 234.52 & 1.12 \\
\hline SurvCI & $(0.759,0.751)$ & $\mathbf{0 . 2 0}$ & $\mathbf{2 3 3 . 9 6}$ & 1.3 \\
\hline \hline Method & $\mathrm{C}_{I}^{(a=0, a=1)}$ & $\epsilon_{A T E}$ & $\sqrt{\epsilon_{P E H E}}$ & $\mathrm{HR}(a)$ \\
\hline DSM & $(0.822,0.731)$ & - & - & 1.47 \\
\hline CRFNet & $(0.624,0.562)$ & 10.37 & 343.93 & - \\
\hline CSA & $(0.787, \mathbf{0 . 7 6 7})$ & 0.80 & 358.72 & 0.45 \\
\hline$\kappa$-SurvCI & $(\mathbf{0 . 8 2 6}, 0.742)$ & 65.23 & 318.751 & 0.63 \\
\hline SurvCI & $(0.805,0.746)$ & $\mathbf{0 . 3 8}$ & $\mathbf{3 0 2 . 7 5}$ & 0.81 \\
\hline
\end{tabular}

TABLE III

PERFormanCE Comparison ON SYNTHETIC (TOP) AND ACTG SEMI-SYNTHETIC DATASET (BOTTOM) WITH RANDOM CENSORING.

\begin{tabular}{|c|c|c|c|c|}
\hline Method & $\mathrm{C}_{I}^{(a=0, a=1)}$ & $\mathrm{C}_{I}^{(a=0, a=1)}$ & $\mathrm{HR}(\mathrm{a})$ & $\mathrm{HR}(\mathrm{a})$ \\
& $\mathrm{RT}$ & $\mathrm{CT}$ & $\mathrm{RT}$ & $\mathrm{CT}$ \\
\hline \hline DSM & $(0.595,0.589)$ & $(0.462,0.637)$ & 0.91 & 0.80 \\
\hline CRFNet & $(0.497,0.576)$ & $(0.572,0.621)$ & - & - \\
\hline TARNet & $(0.409,0.479)$ & $(0.520,0.386)$ & - & - \\
\hline CSA & $(0.599,0.565)$ & $(0.581,0.604)$ & 1.19 & 1.22 \\
\hline$\kappa$-SurvCI & $(0.630,0.635)$ & $(0.504,0.642)$ & 0.93 & 0.93 \\
\hline SurvCI & $(0.635,0.632)$ & $(0.503,0.643)$ & 0.95 & 0.87 \\
\hline SurvCI-Info & $\mathbf{( 0 . 7 7 7 , 0 . 8 0 8})$ & $\mathbf{( 0 . 7 5 , 0 . 7 6 1 )}$ & 1.08 & 2.04 \\
\hline CSA-Info & $(0.557,0.604)$ & $(0.56,0.593)$ & 1.01 & 0.88 \\
\hline
\end{tabular}

TABLE IV

Performance Comparison on the Metabric Dataset

\section{B. Baselines}

We compare the performance of $\kappa$-SurvCI, which is the SurvCI model without the IPM constraint, SurvCI and SurvCI-Info, with the following baselines: 


\begin{tabular}{|c|c|c|c|c|c|c|}
\hline Method & $\mathrm{C}_{I}^{(a=0, a=1)}$ & $\epsilon_{A T E}\left(Y_{T}\right)$ & $\epsilon_{A T E}(Y)$ & $\sqrt{\epsilon_{P E H E}}\left(Y_{T}\right)$ & $\sqrt{\epsilon_{P E H E}}(Y)$ & $\operatorname{HR}(a)$ \\
\hline \hline CSA & $(0.71,0.573)$ & 33.41 & 35.07 & 1129.08 & 715.59 & 0.95 \\
\hline SurvCI & $(0.71,0.62)$ & 124.07 & 124.33 & 1011.43 & 507.43 & 0.96 \\
\hline SurvCI-Info & $\mathbf{( 0 . 7 7 4 , 0 . 7 8 5})$ & $\mathbf{6 . 9 0}$ & $\mathbf{1 3 . 9 0}$ & $\mathbf{1 0 0 2}$ & $\mathbf{4 9 5 . 4 6}$ & 1.07 \\
\hline CSA-Info & $((0.71,0.573))$ & 32.42 & 43.11 & 1071.29 & 567.88 & 0.90 \\
\hline Method & $\mathrm{C}_{I}^{(a=0, a=1)}$ & $\epsilon_{A T E}\left(Y_{T}\right)$ & $\epsilon_{A T E}(Y)$ & $\sqrt{\epsilon_{P E H E}}\left(Y_{T}\right)$ & $\sqrt{\epsilon_{P E H E}}(Y)$ & $\mathrm{HR}(a)$ \\
\hline \hline CSA & $(0.81,0.732)$ & 77.13 & 117.2 & 354.66 & 483.06 & 0.97 \\
\hline SurvCI & $\mathbf{( 0 . 8 2 7 , 0 . 7 3 3 )}$ & 31 & 36.11 & $\mathbf{3 1 5 . 0 2}$ & $\mathbf{3 2 5 . 8 4}$ & 1.01 \\
\hline SurvCI-Info & $(0.80,0.69)$ & 89.16 & $\mathbf{3 . 1 4 6}$ & 370.78 & 464.31 & 0.88 \\
\hline CSA-Info & $(0.812,0.744)$ & 121.36 & 8.11 & 360.87 & 395.61 & 1.01 \\
\hline
\end{tabular}

TABLE V

PERFORMANCE COMPARISON ON SYNTHETIC (TOP) AND ACTG SEMI-SYNTHETIC DATASET (BOTTOM) WITH INFORMATIVE CENSORING.
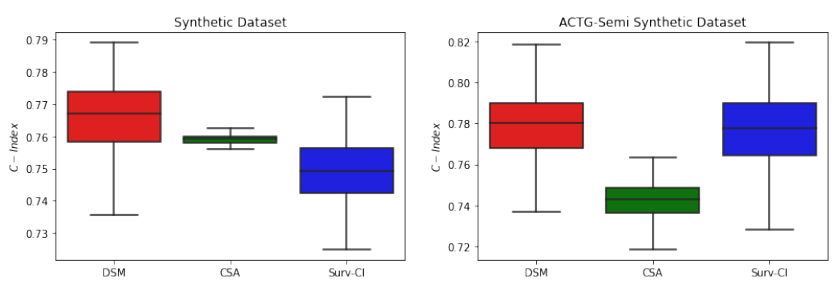

Fig. 4. Box Plot depicting $\mathbf{9 5 \%}$ confidence interval in C-Index for synthetic and ACTG Semi-synthetic Dataset
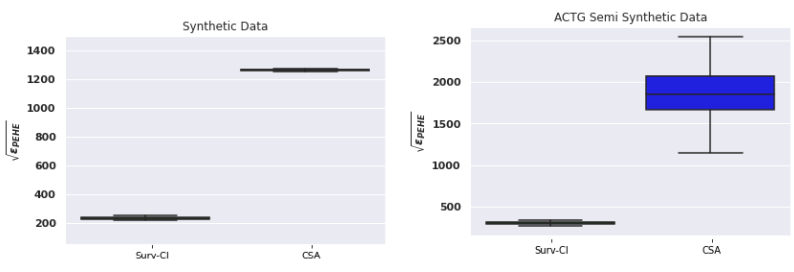

Fig. 5. Box Plot depicting $\mathbf{9 5 \%}$ confidence interval in $\boldsymbol{\epsilon}_{\boldsymbol{P E} \boldsymbol{H} \boldsymbol{E}}$ for synthetic and ACTG semi-synthetic datasets

- DSM [12]: DSM is a CT-SA technique. It does not cater to binary treatments and informative censoring. It outperforms other benchmarks for SA such as Deephit, Deepsurv, CoxPH and AFT models, and this justifies the choice of this technique as a benchmark.

- CFRNet [6]: CFRNet is a technique for counterfactual inference, which not optimized to handle time-to-event outcomes. We use this baseline to demonstrate the efficacy of SurvCI since it explicitly models time-to-event. We also use TARNet baseline, by setting $\kappa=0$ in (8).

- CSA [33]: CSA is a state-of-the-art counterfactual CT-SA technique for observational datasets.

Note that we employ the above baselines as all of them are continuous-time SA techniques. In Table II, we summarize the details of the datasets, where $D$ represents the number of features, $|\mathcal{D}|_{C}$ is the number of censored and $|\mathcal{D}|_{U}$ be the number of time-to-event samples. Further, $N_{1}$ and $N_{0}$ represent the samples with treatment 1 and 0 , respectively. Hence, $|\mathcal{D}|_{C}+|\mathcal{D}|_{U}=N_{1}+N_{0}=N$, where $N$ represents the total number of samples.

\section{Performance Metrics}

We describe the causal and survival metrics used for evaluation of the proposed models [36].
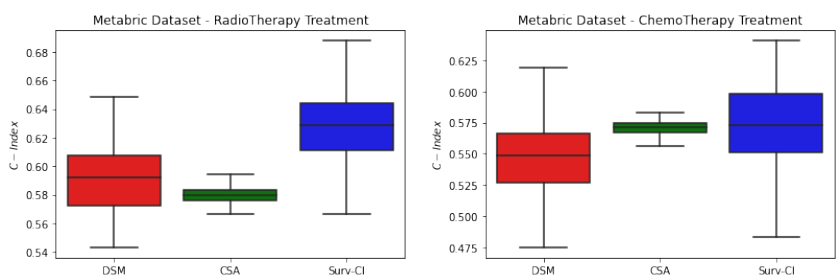

Fig. 6. Metabric: Box Plot depicting $\mathbf{9 5 \%}$ confidence interval in C-Index

Causal Metrics: We compute the estimated error in ATE $\left(\epsilon_{A T E}\right)$ given as $\epsilon_{A T E}=\left|A T E_{i}-A \hat{T} E_{i}\right|=\mid \mathbb{E}_{X}\left[\tau\left(\mathbf{x}_{i}\right)-\right.$ $\left.\hat{\tau}\left(\mathbf{x}_{i}\right)\right] \mid$, where $\tau(\mathbf{x})$ is the ground truth ITE and $\hat{\tau}(\mathbf{x})$ is the predicted ITE, based on RMST. We also report the estimated error in PEHE $\left(\epsilon_{P E H E}\right)$ using (7). In the informative censoring case, unlike the ATE computation in [36], RMST based ATE computation includes time-to-censoring as well. Hence, in these scenarios, we report ATE on $Y_{T}$ and $Y$, where $Y$ consists of $Y_{T}$ and $Y_{C}$.

Survival Metrics: In this work, we compute per-treatment Harrell's C-Index, which is a widely used discriminative index [43]. We also use hazard ratio for comparing the treatment effects on different patient groups [44].

\section{Results and Discussions}

In this section we demonstrate the performance of the proposed SurvCI and SurvCI-Info models and compare it with the benchmarks described in the previous section.

1) Synthetic and ACTG semi-synthetic dataset: We report the survival and causal metrics for the synthetic dataset (top) and ACTG semi-synthetic dataset (bottom) in Table III, in the presence of non-informative censoring. In terms of the $\mathrm{C}$ Index, the proposed technique is comparable to DSM. This is expected since the representation layer is trained to abate the confounding bias, and SA per treatment is a part of the hypothesis layer. We observe that all of the proposed models outperform CFRNet with respect to causal metrics. Considering causal metrics, we see that the proposed techniques outperform all the benchmarks, and in particular, outperforms CSA, the state-of-the-art benchmark. Our method performs especially well in terms of $\epsilon_{P E H E}$ metric, mainly due to the use of RMST as a part of the squared error loss which ensures that we minimize the variance in the survival time. Similar trends are observed for the ACTG dataset, where 
most of the methods are comparable w.r.t C-Index, but SurvCI performs the best w.r.t causal metrics. The effect of treatment (probabilistic) is seen from $H R(a)$. We see that DSM, which ignores the effect of selection bias, favorably or unfavorably overemphasizes $H R(a)$. However, techniques that compensate for the selection bias have a more realistic estimate of $H R(a)$.

We report the causal and survival metrics of SurvCI-Info and SurvCI on datasets with informative censoring - synthetic and semi-synthetic ACTG datasets in Tab. V. The causal metrics are reported both, for $Y_{T}$ and $Y$, where $Y$ consists of both $Y_{T}$ and $Y_{C}$. It can be seen that the PEHE error for all the methods are lower for $Y$ as compared to estimation of $Y_{T}$ which implies that obtaining PEHE error on $Y$ is more reliable. We see that SurvCI-Info outperforms all the baselines including CSA-Info with respect $\epsilon_{A T E}$ on $Y$, while all the methods have comparable C-Index performance. However, SurvCI performs the best w.r.t the PEHE metric for both, $Y_{T}$ and $Y$.

Box-plots: We present the $95 \%$ percentile box plots for CIndex and $\epsilon_{\text {PEHE }}$, for the synthetic, semi-synthetic datasets with non-informative censoring, and the real-world Metabic datasets. We see that C-Index of the proposed SurvCI is comparable or better to DSM and CSA for ACTG and Metabric dataset (RT). However, SurvCI consistently performs better than CSA with respect to $\epsilon_{\mathrm{PEHE}}$ metric in the presence of noninformative censoring.

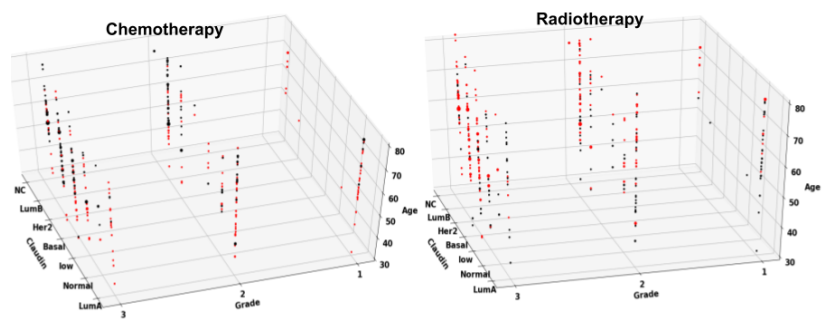

Fig. 7. Metabric: Multi-dimensional plots depicting the features of patients with $\operatorname{HR}(a)<\mathbf{1}$ (black) and $>\mathbf{1}$ (red). The size of each point is proportional to the number of positive lymph nodes for that patient.

2) Metabric dataset: In the Metabric dataset, the time-toevent is the survival months, which is time-to-death. Here, $\operatorname{HR}(a)<1$ is preferred since the instantaneous probability of death with $a=1$ is lower than $a=0$, i.e., the treatment is beneficial. We consider RT and CT as the two treatments, and analyse the distribution of patients for $\operatorname{HR}(a)<1$ (in blue, Fig. 7), as compared to the entire population (in green, Fig. 7). We observed that the maximum variation of the distribution occurred for the features depicted, namely Lymph nodes examined positive, age, PAM50+Claudin subtype and histological grade. We deduce that by analysing the interplay between these features, a better understanding can be developed about treatment effectiveness.

For any real-world dataset, we do not have the counterfactuals, and hence, we report the survival metrics. From Table. IV, SurvCI and $\kappa$-SurvCI reported an improved C-Index as compared to baseline C-Index of DSM and CSA. Benchmarks such as TARNet and CFRNet were not able to learn the model well, and reported a C-Index $<0.5$. However, SurvCI-Info obtains the best model w.r.t C-Index over both the treatments. We calculated $\operatorname{HR}(a)$ for the test samples and we obtained overall $\operatorname{HR}(a) \sim 1$ for SurvCI and its variants.

In Fig. 7, we depict the 5-D plot where the individuals with $\operatorname{HR}(a)<1$ are shown in black, and $\operatorname{HR}(a)>1$ in red, for RT and CT. The axes correspond to age, grade of BC (1,2 or 3$)$ and cancer sub-type (feature called Claudin), and the size of the points is proportional to the number of positive lymph nodes. We observed that these features have a different histogram for $\operatorname{HR}(a)<1$, as compared to the overall population [45]. Typically $\mathrm{BC}$ is profiled using molecular subtypes namely luminal A, luminal B, HER2-enriched and basal-like [46]. Each of these have different treatment response profiles. In Fig. 7 for CT, we see that the treatment is effective on the luminal A subtype, for younger patients with fewer positive lymph nodes. CT is known to be less effective for luminal B subtype [47]. We also observe that CT is marginally effective for luminal A subtype with small number of lymph nodes being positive [48]. RT is generally used post-operative and is effective for all subtypes and grades [49] as seen from Fig. 7.

\section{CONCLUSIONS AND FUTURE WORK}

We proposed the novel SurvCI and SurvCI-Info approaches for CI on SA datasets with random and instancedependent informative censoring, respectively. We derived the parametric mixture-model based ELBO framework for SA in the presence of binary treatments and non-informative or informative censoring. We used the balanced representation framework for CI, and showed that SA is incorporated into CI using an RMST based loss function. In the informative censoring case, we modeled both instance-dependent time-to-censoring along with time-to-event, and derived the SurvCI-Info model. We demonstrated the performance of the model on a novel synthetic, ACTG semi-synthetic dataset [36] and Metabric dataset. We showed that SurvCI and SurvCI-Info models outperform baseline methods significantly with respect to the causal metrics. For the realworld dataset, we computed the survival metrics and stated some interesting observations. Future works include extending our approach to the competing risks and multiple treatments and dosages scenarios.

\section{References}

[1] Samir M Hanash, Christina S Baik, and Olli Kallioniemi, "Emerging molecular biomarkers-blood-based strategies to detect and monitor cancer," Nature reviews Clinical oncology, vol. 8, no. 3, pp. 142-150, 2011.

[2] Heidi Seibold, Achim Zeileis, and Torsten Hothorn, "Model-based recursive partitioning for subgroup analyses," The international journal of biostatistics, vol. 12, no. 1, pp. 45-63, 2016.

[3] Sachin Kumar, Garima Gupta, Ranjitha Prasad, Arnab Chatterjee, Lovekesh Vig, and Gautam Shroff, "Camta: Causal attention model for multi-touch attribution," in 2020 International Conference on Data Mining Workshops (ICDMW). IEEE, 2020, pp. 79-86.

[4] Léon Bottou, Jonas Peters, Joaquin Quiñonero-Candela, Denis X Charles, D Max Chickering, Elon Portugaly, Dipankar Ray, Patrice Simard, and Ed Snelson, "Counterfactual reasoning and learning systems: The example of computational advertising.," Journal of Machine Learning Research, vol. 14, no. 11, 2013.

[5] Donald B Rubin, "Estimating causal effects of treatments in randomized and nonrandomized studies.," Journal of educational Psychology, vol. 66, no. 5, pp. 688, 1974. 
[6] Uri Shalit, Fredrik D Johansson, and David Sontag, "Estimating individual treatment effect: generalization bounds and algorithms," in International Conference on Machine Learning. PMLR, 2017, pp. 30763085.

[7] Weijia Zhang, Jiuyong Li, and Lin Liu, "A unified survey of treatment effect heterogeneity modelling and uplift modelling," ACM Computing Surveys (CSUR), vol. 54, no. 8, pp. 1-36, 2021.

[8] Liuyi Yao, Zhixuan Chu, Sheng Li, Yaliang Li, Jing Gao, and Aidong Zhang, "A survey on causal inference," arXiv preprint arXiv:2002.02770, 2020.

[9] Saharon Rosset, Einat Neumann, Uri Eick, Nurit Vatnik, Hapnina St, and Yizhak Idan, "Customer lifetime value modeling and its use for customer retention planning," 082002.

[10] Jerald F Lawless, Statistical models and methods for lifetime data, vol. 362, John Wiley \& Sons, 2011

[11] Ping Wang, Yan $\mathrm{Li}$, and Chandan K Reddy, "Machine learning for survival analysis: A survey," ACM Computing Surveys (CSUR), vol. 51 , no. 6, pp. 1-36, 2019

[12] Chirag Nagpal, Xinyu Rachel Li, and Artur Dubrawski, "Deep survival machines: Fully parametric survival regression and representation learning for censored data with competing risks," IEEE Journal of Biomedical and Health Informatics, 2021.

[13] John Fox and Sanford Weisberg, "Cox proportional-hazards regression for survival data," An R and S-PLUS companion to applied regression, vol. 2002, 2002

[14] Lee-Jen Wei, "The accelerated failure time model: a useful alternative to the cox regression model in survival analysis," Statistics in medicine, vol. 11, no. 14-15, pp. 1871-1879, 1992.

[15] Jared L Katzman, Uri Shaham, Alexander Cloninger, Jonathan Bates, Tingting Jiang, and Yuval Kluger, "Deepsurv: personalized treatment recommender system using a cox proportional hazards deep neural network," BMC medical research methodology, vol. 18, no. 1, pp. 1-12, 2018.

[16] Margaux Luck, Tristan Sylvain, Héloïse Cardinal, Andrea Lodi, and Yoshua Bengio, "Deep learning for patient-specific kidney graft survival analysis," arXiv preprint arXiv:1705.10245, 2017.

[17] Matthew Pawley, "Deepweibull: a deep learning approach to parametric survival analysis," 2020.

[18] Rodney Sparapani, Brent R Logan, Robert E McCulloch, and Purushottam W Laud, "Nonparametric competing risks analysis using bayesian additive regression trees," Statistical methods in medical research, vol. 29, no. 1, pp. 57-77, 2020.

[19] Changhee Lee, William R Zame, Jinsung Yoon, and Mihaela van der Schaar, "Deephit: A deep learning approach to survival analysis with competing risks," in Thirty-second AAAI conference on artificial intelligence, 2018.

[20] Kwan-Moon Leung, Robert M. Elashoff, and Abdelmonem A. Afifi, "Censoring issues in survival analysis," Annual Review of Public Health, vol. 18, no. 1, pp. 83-104, 1997, PMID: 9143713.

[21] Stephen W Lagakos, "General right censoring and its impact on the analysis of survival data," Biometrics, pp. 139-156, 1979.

[22] Christos Louizos, Uri Shalit, Joris Mooij, David Sontag, Richard Zemel, and Max Welling, "Causal effect inference with deep latent-variable models," arXiv preprint arXiv:1705.08821, 2017.

[23] Ioana Bica, James Jordon, and Mihaela van der Schaar, "Estimating the effects of continuous-valued interventions using generative adversarial networks," in NeurIPS, H. Larochelle, M. Ranzato, R. Hadsell, M. F. Balcan, and H. Lin, Eds., 2020, vol. 33, pp. 16434-16445.

[24] Ahmed M Alaa and Mihaela van der Schaar, "Bayesian inference of individualized treatment effects using multi-task gaussian processes," arXiv preprint arXiv:1704.02801, 2017.

[25] Jinsung Yoon, James Jordon, and Mihaela Van Der Schaar, "Ganite: Estimation of individualized treatment effects using generative adversarial nets," in International Conference on Learning Representations, 2018.

[26] Fredrik Johansson, Uri Shalit, and David Sontag, "Learning representations for counterfactual inference," in International conference on machine learning. PMLR, 2016, pp. 3020-3029.

[27] Ankit Sharma, Garima Gupta, Ranjitha Prasad, Arnab Chatterjee, Lovekesh Vig, and Gautam Shroff, "Metaci: Meta-learning for causal inference in a heterogeneous population," arXiv preprint arXiv: 1912.03960, 2019.

[28] Ya Li, Xinmei Tian, Mingming Gong, Yajing Liu, Tongliang Liu, Kun Zhang, and Dacheng Tao, "Deep domain generalization via conditional invariant adversarial networks," in Proceedings of the European Conference on Computer Vision (ECCV), 2018, pp. 624-639.
[29] Per K Andersen, Elisavet Syriopoulou, and Erik T Parner, "Causal inference in survival analysis using pseudo-observations," Statistics in medicine, vol. 36, no. 17, pp. 2669-2681, 2017.

[30] Ariel Linden and Paul R Yarnold, "Estimating causal effects for survival (time-to-event) outcomes by combining classification tree analysis and propensity score weighting," Journal of Evaluation in Clinical Practice, vol. 24, no. 2, pp. 380-387, 2018

[31] Yifan Cui, Michael R Kosorok, Erik Sverdrup, Stefan Wager, and Ruoqing Zhu, "Estimating heterogeneous treatment effects with right-censored data via causal survival forests," arXiv preprint arXiv:2001.09887, 2020.

[32] Jincheng Shen, Lu Wang, Stephanie Daignault, Daniel E Spratt, Todd M Morgan, and Jeremy MG Taylor, "Estimating the optimal personalized treatment strategy based on selected variables to prolong survival via random survival forest with weighted bootstrap," Journal of biopharmaceutical statistics, vol. 28, no. 2, pp. 362-381, 2018.

[33] Paidamoyo Chapfuwa, Serge Assaad, Shuxi Zeng, Michael J Pencina, Lawrence Carin, and Ricardo Henao, "Enabling counterfactual survival analysis with balanced representations," in Proceedings of the Conference on Health, Inference, and Learning, 2021, pp. 133-145.

[34] Alicia Curth, Changhee Lee, and Mihaela van der Schaar, "Survite: Learning heterogeneous treatment effects from time-to-event data," Advances in Neural Information Processing Systems, vol. 34, 2021.

[35] Paidamoyo Chapfuwa, Chenyang Tao, Chunyuan Li, Courtney Page, Benjamin Goldstein, Lawrence Carin Duke, and Ricardo Henao, "Adversarial time-to-event modeling," in ICML. 10-15 Jul 2018, vol. 80 of Proceedings of Machine Learning Research, pp. 735-744, PMLR.

[36] Paidamoyo Chapfuwa, Chenyang Tao, Chunyuan Li, Courtney Page, Benjamin Goldstein, Lawrence Carin Duke, and Ricardo Henao, "Adversarial time-to-event modeling," in International Conference on Machine Learning. PMLR, 2018, pp. 735-744.

[37] Paul R Rosenbaum and Donald B Rubin, "The central role of the propensity score in observational studies for causal effects," Biometrika vol. 70, no. 1, pp. 41-55, 1983.

[38] Guido W Imbens and Donald B Rubin, Causal inference in statistics, social, and biomedical sciences, Cambridge University Press, 2015.

[39] Arthur Gretton, Karsten M Borgwardt, Malte J Rasch, Bernhard Schölkopf, and Alexander Smola, "A kernel two-sample test," The Journal of Machine Learning Research, vol. 13, no. 1, pp. 723-773, 2012.

[40] David M Zucker, "Restricted mean life with covariates: modification and extension of a useful survival analysis method," Journal of the American Statistical Association, vol. 93, no. 442, pp. 702-709, 1998.

[41] Yaru Liang, Chang Liu, Maoyang Lu, Qiongye Dong, Zimu Wang, Zhuoran Wang, Wenxiang Xiong, Nannan Zhang, Jiawei Zhou, Qingfei Liu, et al., "Calorie restriction is the most reasonable anti-ageing intervention: a meta-analysis of survival curves," Scientific reports, vol. 8, no. 1, pp. 1-9, 2018.

[42] Marlene Smurzynski, Ann C Collier, Susan L Koletar, Ronald J Bosch, Kunling Wu, Barbara Bastow, and Constance A Benson, "Aids clinical trials group longitudinal linked randomized trials (allrt): rationale, design, and baseline characteristics," HIV clinical trials, vol. 9, no. 4, pp. 269-282, 2008

[43] F. E. Jr Harrell, K. L. Lee, R. M. Califf, D.B. Pryor, and Rosati R. A., "Regression modelling strategies for improved prognostic prediction," Stat. Med., vol. 3(2), pp. 143-52, 1984.

[44] Spotswood L Spruance, Julia E Reid, Michael Grace, and Matthew Samore, "Hazard ratio in clinical trials," Antimicrobial agents and chemotherapy, vol. 48, no. 8, pp. 2787-2792, 2004.

[45] Chen Y., Jia Z., Mercola D., and Xie X., "A gradient boosting algorithm for survival analysis via direct optimization of concordance index," Comput Math Methods Med., 2013.

[46] X. Dai, T. Li, Z. Bai, Y. Yang, X. Liu, J. Zhan, and B. Shi, "Breast cancer intrinsic subtype classification, clinical use and future trends," vol. 5(10), pp. 2929-2943, 2015

[47] Z. H. Li, J. H. Hu, P. H.and Tu, and N. S. Yu, "Luminal B breast cancer: patterns of recurrence and clinical outcome," Oncotarget, vol. 7(40), pp. 65024-65033, 2016.

[48] N. Uchida, T. Suda, and K. Ishiguro, "Effect of chemotherapy for Luminal-A breast cancer," ESANN, vol. 56(2), pp. 51-56, 2013.

[49] F.E. Langlands, K. Horgan, D.D. Dodwell, and Smith L., "Breast cancer subtypes: response to radiotherapy and potential radiosensitisation.," $\mathrm{Br}$ J Radiol, vol. 86, 2013. 